TRANSACTIONS OF THE

AMERICAN MATHEMATICAL SOCIETY

Volume 358, Number 8, Pages 3263-3283

S 0002-9947(06)04042-6

Article electronically published on March 24, 2006

\title{
LOWER AND UPPER LOEB-INTEGRALS
}

\author{
D. LANDERS AND L. ROGGE
}

\begin{abstract}
We introduce the concepts of lower and upper Loeb-integrals for an internal integration structure. These are concepts which are similarly useful for Loebs internal integration theory as the concepts of inner and outer Loebmeasures for Loebs measure theory.
\end{abstract}

\section{INTRODUCTION AND NOTATION}

Almost the whole nonstandard measure and probability theory is based on fundamental concepts and results of Loeb. Starting from an internal content, Loeb constructed in particular an important standard measure, called Loeb-measure in the literature (see [8], 9]). This Loeb-measure has been investigated and applied in many papers by Loeb and other authors to obtain new results in various fields of mathematics such as e.g. in mathematical physics and economics, in measure and probability theory and in potential theory. Loeb extended his measure theoretical approach to integration theory and introduced the so-called Loeb-integrals (see [10], [11).

To construct the Loeb-measure, Loeb used the Carathéodory extension theorem. Another construction of the Loeb-measure for finite internal contents can be given in terms of the inner and outer Loeb-measures. Sommers (see [13]) investigated inner Loeb-measures also for nonfinite internal contents.

Inner and outer Loeb-measures are very powerful concepts that can be used in many situations. Inner and outer Loeb-measures have been applied e.g. in the construction of Radon measures and $\tau$-smooth measures, in the extension of $\tau$-smooth Baire or Radon-Baire measures to $\tau$-smooth Borel or Radon-Borel measures, or in compactness criteria for families of probability measures with respect to the weak topology (see [5], 6]).

To develop the Loeb integration theory for internal integration structures we introduce a lower and an upper Loeb-integral; concepts which are of comparable interest and usefulness as inner and outer Loeb-measures. A similar concept of lower Loeb-integrals can be found in Aldaz (see 1]).

In the following we consider a sufficiently rich superstructure $\widehat{S}$ and work with a nonstandard model for this superstructure, which is polysaturated, i.e. if $\mathcal{C}$ is a system of internal sets with cardinality $|\mathcal{C}|$ smaller than or equal to the cardinality $|\widehat{S}|$, then we have $\bigcap_{C \in \mathcal{C}} C \neq \emptyset$ if $\mathcal{C}$ has the finite intersection property. For the general theory of nonstandard-analysis see the books of Cutland [2], Hurd-Loeb [4]

Received by the editors October 5, 2001.

2000 Mathematics Subject Classification. Primary 28E05; Secondary 26E35.

Key words and phrases. Loeb-measure, Loeb-integral, $\tau$-continuity.

(C)2006 American Mathematical Society

Reverts to public domain 28 years from publication 
or [7. Let $\operatorname{fin}\left({ }^{*} \mathbb{R}\right)$ be the set of finite numbers of ${ }^{*} \mathbb{R}$. For $a \in \operatorname{fin}\left({ }^{*} \mathbb{R}\right),{ }^{\circ} a$ denotes the standard number nearest to $a$ in $\mathbb{R}$. If $a \in{ }^{*} \mathbb{R}$ is negative (positive) infinite put ${ }^{\circ} a=-\infty(\infty)$.

In the following, let $\mathcal{E} \subset{ }^{*} \mathbb{R}^{Y}$ be an internal Stonian lattice and let $i: \mathcal{E} \rightarrow{ }^{*} \mathbb{R}$ be an internal, positive linear functional, i.e. $i$ is internal and for all $e, e_{1}, e_{2} \in \mathcal{E}$ and $\alpha_{1}, \alpha_{2} \in{ }^{*} \mathbb{R}$

$$
\begin{gathered}
|e| \in \mathcal{E}, 1 \wedge e \in \mathcal{E} \text { and } \alpha_{1} e_{1}+\alpha_{2} e_{2} \in \mathcal{E} \\
i\left(\alpha_{1} e_{1}+\alpha_{2} e_{2}\right)=\alpha_{1} i\left(e_{1}\right)+\alpha_{2} i\left(e_{2}\right) \\
e \geq 0 \Rightarrow i(e) \geq 0
\end{gathered}
$$

We call $(\mathcal{E}, i)$ an internal integration structure on $Y$ (see Hurd-Loeb [4, pp. 166-168). Let

$$
\mathcal{E}^{f i n}:=\left\{e \in \mathcal{E}: i(|e|) \in \operatorname{fin}\left({ }^{*} \mathbb{R}\right)\right\}
$$

and put, for $g: Y \rightarrow{ }^{*} \mathbb{R}$,

$$
\begin{aligned}
& \underline{i}(g):=\sup \left\{{ }^{\circ} i(e): \mathcal{E}^{f i n} \ni e \leq g\right\} \\
& \bar{i}(g):=\inf \left\{{ }^{\circ} i(e): g \leq e \in \mathcal{E}^{f i n}\right\} \\
& \mathcal{L}(i):=\left\{f \in \mathbb{R}^{Y}: \underline{i}(f)=\bar{i}(f) \in \mathbb{R}\right\} .
\end{aligned}
$$

$\underline{i}$ is called the lower Loeb-integral, $\bar{i}$ the upper Loeb-integral and $\mathcal{L}(i)$ the system of Loeb-integrable functions. If $f \in \mathcal{L}(i)$, we write $i^{L}(f)$ instead of $\underline{i}(f)(=\bar{i}(f))$, and call $i^{L}(f)$ the Loeb-integral of $f$.

Let $\rho: \mathcal{P}(Y) \rightarrow[0, \infty]$ be a monotone function with $\rho(\emptyset)=0$. Then it is well known that $\mathcal{M}(\rho):=\{M \subset Y: \forall A \in \mathcal{P}(Y)(\rho(A)=\rho(A \cap M)+\rho(A \backslash M))\}$ is an algebra and $\rho$ is an additive function on $\mathcal{M}(\rho)$. We apply this result in the following to the set functions $\rho=\underline{i}$ or $\rho=\bar{i}$, where $\underline{i}(A):=\underline{i}\left(1_{A}\right), \bar{i}(A):=\bar{i}\left(1_{A}\right)$ for $A \in \mathcal{P}(Y)$. Furthermore

$$
\mathcal{M}_{0}:=\left\{A \in \mathcal{P}(Y): 1_{A} \in \mathcal{L}(i)\right\}=\{A \in \mathcal{P}(Y): \underline{i}(A)=\bar{i}(A) \in \mathbb{R}\}
$$

is called the system of Loeb-integrable sets. For $M_{0} \in \mathcal{M}_{0}$ we also write $i^{L}\left(M_{0}\right)$ instead of $\underline{i}\left(M_{0}\right)\left(=\bar{i}\left(M_{0}\right)\right)$. The systems $\mathcal{M}(\underline{i})$, respectively $\mathcal{M}(\bar{i})$, are called the system of lower Loeb-measurable sets, respectively upper Loeb-measurable sets.

\section{The MAIN RESUlts}

The following theorem shows that our system $\mathcal{L}(i)$ is the same system as introduced by Loeb in [10, [11] and [4].

1. Theorem. For a function $f: Y \rightarrow \mathbb{R}$ the following are equivalent:

(i) $f \in \mathcal{L}(i)$;

(ii) there exist $e_{n} \in \mathcal{E}^{\text {fin }}$ with $\bar{i}\left(\left|f-e_{n}\right|\right) \underset{n \rightarrow \infty}{\rightarrow} 0$;

(iii) there exists $e \in \mathcal{E}^{f i n}$ with $\bar{i}(|f-e|)=0$.

If $e_{n}$ is a sequence fulfilling (ii), then $i^{L}(f)=\lim _{n \rightarrow \infty}{ }^{\circ} i\left(e_{n}\right)$; if $e$ is a function fulfilling (iii), then $i^{L}(f)={ }^{\circ} i(e)$.

Let us remark that even for the formulation of Theorem 1 it is essential that we have defined the upper Loeb-integral $\bar{i}$ for functions with values in ${ }^{*} \mathbb{R}$ and not only for functions with values in $\mathbb{R}$. 
Most of the parts of the following theorem are due to Loeb (see [10, 4]). The proofs given here are different and use the properties of the inner and outer Loebintegral.

2. Theorem. (i) $i^{L}: \mathcal{L}(i) \rightarrow \mathbb{R}$ is a positive linear functional on the Stonian lattice $\mathcal{L}(i)$.

(ii) $\mathcal{L}(i) \ni f_{n} \uparrow f \in \mathbb{R}^{Y}$ and $\sup _{n \in \mathbb{N}} i^{L}\left(f_{n}\right)<\infty$ imply $f \in \mathcal{L}(i)$ and $i^{L}\left(f_{n}\right) \uparrow i^{L}(f)$.

(iii) $f, h \in \mathcal{L}(i)$ and $f \leq g \leq h$ with $i^{L}(f)=i^{L}(h)$ imply $g \in \mathcal{L}(i)$.

The following theorem shows that the Loeb-integral $i^{L}$ on $\mathcal{L}(i)$ is in fact a classical Lebesgue-integral with respect to the measure space $(Y, \mathcal{M}(\bar{i}), \bar{i})$.

3. Theorem. (i) $\bar{i}$ is a complete and saturated measure on the $\sigma$-algebra $\mathcal{M}(\bar{i})$.

(ii) $\mathcal{M}(\bar{i})=\left\{A \subset Y:\left(\forall M_{0} \in \mathcal{M}_{0}\right)\left(A \cap M_{0} \in \mathcal{M}_{0}\right)\right\}$.

(iii) $\mathcal{M}_{0}$ is a $\delta$-ring and $\mathcal{M}_{0}=\{A \in \mathcal{M}(\bar{i}): \bar{i}(A) \in \mathbb{R}\}$.

(iv) $\bar{i}(f)=\int f d \bar{i}$ for each $\mathcal{M}(\bar{i})$-measurable function $f: Y \rightarrow[0, \infty[$.

(v) $\mathcal{L}(i)=\left\{f \in \mathbb{R}^{Y}: f\right.$ is $\bar{i} \mid \mathcal{M}(\bar{i})$-Lebesgue-integrable $\}$ and $i^{L}(f)=\int f d \bar{i}$ for each $f \in \mathcal{L}(i)$.

(vi) $0 \leq f \in \mathcal{L}(i)$ and $r>0 \Rightarrow\{f>r\} \in \mathcal{M}_{0}$.

In the following theorem we consider the lower Loeb-integral and compare it with the classical Lebesgue-integral with respect to the measure space $(Y, \mathcal{M}(\underline{i}), \underline{i})$. For many problems in stochastics it turns out that the $\sigma$-algebra $\mathcal{M}(\underline{i})$ - which is in general strictly smaller than the $\sigma$-algebra $\mathcal{M}(\bar{i})$ - is the suitable $\sigma$-algebra. The reason is that many important functions are even $\mathcal{M}(\underline{i})$-measurable and not only $\mathcal{M}(\bar{i})$-measurable. Hence we obtain more information about those functions. Examples for such functions are ${ }^{\circ} e$ for $e \in \mathcal{E}$ or the standard part map of regular topological spaces. In all those spaces the measure $\underline{i}$ (and not $\bar{i}$ ) allows us to construct Radon-measures of great interest.

4. Theorem. (i) $\underline{i}$ is a complete measure on the $\sigma$-algebra $\mathcal{M}(\underline{i})$.

(ii) $\mathcal{M}(\underline{i}) \subset \mathcal{M}(\bar{i})$.

(iii) $\mathcal{M}_{0} \subset\{A \in \mathcal{M}(\underline{i}): \underline{i}(A) \in \mathbb{R}\}$.

(iv) $\underline{i}(f)=\int f d \underline{i}$ for each $\mathcal{M}(\underline{i})$-measurable function $f: Y \rightarrow[0, \infty[$.

(v) $\mathcal{L}(i) \subset\left\{f \in \mathbb{R}^{Y}: f\right.$ is $\underline{i} \mid \mathcal{M}(\underline{i})$-Lebesgue-integrable $\}$ and $i^{L}(f)=\int f d \underline{i}$ for each $f \in \mathcal{L}(i)$.

Let us remark (see Example 20 in $\S 3$ ) that it can happen that:

(i) $\underline{i} \mid \mathcal{M}(\underline{i})$ is not a saturated measure;

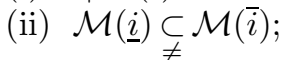

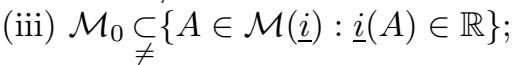

(iv) $\mathcal{L}(i) \underset{\neq}{\subset}\left\{f \in \mathbb{R}^{Y}: f\right.$ is $\underline{i} \mid \mathcal{M}(\underline{i})$-Lebesgue-integrable $\}$.

If $f: Y \rightarrow[0, \infty]$ is $\mathcal{M}(\underline{i})$-, respectively $\mathcal{M}(\bar{i})$-, measurable, then we write $\underline{i}(f)$, respectively $\bar{i}(f)$, for $\int f d \underline{i}$, respectively $\int f d \bar{i}$. This notation is justified by Theorem 3 (iv), respectively Theorem 4 (iv), for real-valued functions. Observe that for a function $f: Y \rightarrow[0, \infty]$ which attains the value $\infty, \underline{i}(f)$ and $\bar{i}(f)$ were not defined before.

The following example shows that internal contents lead in a natural way to upper and lower Loeb-integrals which coincide on sets with the inner and outer Loeb-measures. 
If $\nu: \mathcal{R} \rightarrow{ }^{*}[0, \infty[$ is an internal content on a ring $\mathcal{R} \subset \mathcal{P}(Y)$, then

$$
\begin{aligned}
\underline{\nu}(A) & :=\sup \left\{{ }^{\circ} \nu(R): \mathcal{R} \ni R \subset A\right\}, \\
\bar{\nu}(A) & :=\inf \left\{{ }^{\circ} \nu(R): A \subset R \in \mathcal{R}\right\}
\end{aligned}
$$

denote the usual inner and outer Loeb-measure of $\nu$.

5. Example. Let $\nu: \mathcal{R} \rightarrow{ }^{*}[0, \infty$ [ be an internal content on a ring $\mathcal{R} \subset P(Y)$. Let $\mathcal{E}$ be the system of all internal sums $\sum_{j=1}^{h} \alpha_{j} 1_{R_{j}}$ with $\alpha_{j} \in{ }^{*} \mathbb{R}, R_{j} \in \mathcal{R}, h \in{ }^{*} \mathbb{N}$ and put

$$
i_{\nu}\left(\sum_{j=1}^{h} \alpha_{j} 1_{R_{j}}\right)=\sum_{j=1}^{h} \alpha_{j} \nu\left(R_{j}\right) .
$$

Then $\mathcal{E}$ is an internal Stonian lattice, $i_{\nu}: \mathcal{E} \rightarrow{ }^{*} \mathbb{R}$ is an internal positive linear functional and we have

(i) $\underline{\nu}(R)=\bar{\nu}(R)={ }^{\circ} \nu(R)$ for all $R \in \mathcal{R}$;

(ii) $\underline{\nu}(A)=\underline{i_{\nu}}(A), \bar{\nu}(A)=\overline{i_{\nu}}(A)$ for all $A \subset Y$;

(iii) $\mathcal{M}_{0}=\{\bar{A} \subset Y: \underline{\nu}(A)=\bar{\nu}(A)<\infty\}$;

(iv) $\mathcal{R} \subset \mathcal{M}(\underline{\nu}) \subset \mathcal{M}(\bar{\nu})$;

(v) $\underline{\nu}$ is a complete measure on the $\sigma$-algebra $\mathcal{M}(\underline{\nu}) ; \bar{\nu}$ is a complete and saturated measure on the $\sigma$-algebra $\mathcal{M}(\bar{\nu})$.

The following theorem generalizes the $\widehat{S}$-continuity of the inner and outer Loebmeasure for finite internal contents (see [5]) to $\widehat{S}$-continuity of inner and outer Loeb-integrals.

6. Theorem. Let $\mathcal{E}_{1} \subset \mathcal{E}$ with cardinality of $\mathcal{E}_{1}$ smaller than or equal to $\widehat{S}$. Then

(i) $\sup ^{\circ} e$ is $\mathcal{M}(\underline{i})$-measurable. $e \in \mathcal{E}_{1}$

If $\mathcal{E}_{1}$ is furthermore an upwards directed system of nonnegative functions, then

(ii) $\sup _{e \in \mathcal{E}_{1}} \bar{i}\left({ }^{\circ} e\right)=\bar{i}\left(\sup _{e \in \mathcal{E}_{1}}{ }^{\circ} e\right)$;

(iii) $\sup _{e \in \mathcal{E}_{1}} \underline{i}\left({ }^{\circ} e\right)=\underline{i}\left(\sup _{e \in \mathcal{E}_{1}}{ }^{\circ} e\right)$;

(iv) $\underline{i}\left(\sup _{e \in \mathcal{E}_{1}}{ }^{\circ} e\right)=\bar{i}\left(\sup _{e \in \mathcal{E}_{1}}{ }^{\circ} e\right)$.

The following two theorems are fundamental for the application of inner and outer Loeb-integrals to obtain similar representation results as were given for finite Loeb-measures in [5]; in these cases $Y_{0}$ is the set $n s^{*} X$ of near-standard points, which is in general not measurable. Applications of the following two theorems will be given in a forthcoming paper.

7. Theorem. Let $\emptyset \neq Y_{0} \subset Y$. Then

(i) $\underline{i}\left|\mathcal{M}(\underline{i}) \cap Y_{0}, \bar{i}\right| \mathcal{M}(\bar{i}) \cap Y_{0}$ are measures;

(ii) $\underline{i}\left(f 1_{Y_{0}}\right)=\left.\left.\int f\right|_{Y_{0}} d \underline{i}\right|_{Y_{0}}$;

(iii) $\bar{i}\left(f 1_{Y_{0}}\right)=\left.\left.\int f\right|_{Y_{0}} d \bar{i}\right|_{Y_{0}}$;

where we assume in (ii), respectively (iii), that the function $f: Y \rightarrow[0, \infty[$ is $\mathcal{M}(\underline{i})$-, respectively $\mathcal{M}(\bar{i})-$, measurable and $\left.\underline{i}\right|_{Y_{0}}$, respectively $\left.\bar{i}\right|_{Y_{0}}$, is the measure $\underline{i} \mid \mathcal{M}(\underline{i}) \cap Y_{0}$, respectively $\bar{i} \mid \mathcal{M}(\bar{i}) \cap Y_{0}$.

If $f: Y \rightarrow[0, \infty]$ is $\mathcal{M}(\underline{i})$-, respectively $\mathcal{M}(\bar{i})$-, measurable, then we write $\underline{i}\left(f 1_{Y_{0}}\right)$, respectively $\bar{i}\left(f 1_{Y_{0}}\right)$, for $\left.\left.\int f\right|_{Y_{0}} d \underline{i}\right|_{Y_{0}}$, respectively $\left.\left.\int f\right|_{Y_{0}} d \bar{i}\right|_{Y_{0}}$. This notation is justified by Theorem 7 . 
8. Theorem. Let $\mathcal{E}_{1} \subset \mathcal{E}$ be an upwards directed system of nonnegative functions with cardinality smaller than or equal to $\widehat{S}$. Then for each $Y_{0} \subset Y$ we have

(i) $\underline{i}\left(\sup _{e \in \mathcal{E}_{1}}{ }^{\circ} e 1_{Y_{0}}\right)=\sup _{e \in \mathcal{E}_{1}} \underline{i}\left({ }^{\circ} e 1_{Y_{0}}\right)$;

(ii) $\bar{i}\left(\sup _{e \in \mathcal{E}_{1}}{ }^{\circ} e 1_{Y_{0}}\right)=\sup _{e \in \mathcal{E}_{1}} \bar{i}\left({ }^{\circ} e 1_{Y_{0}}\right)$;

where we assume in (i) and (ii) that the left-hand terms are finite.

We show in Example 19 in $\S 3$ that the finiteness condition in Theorem 8 (ii) cannot be omitted.

\section{Auxiliary lemmata and proof of the Results}

Proof of Theorem 1. (iii) $\Rightarrow$ (ii) is trivial. (ii) $\Rightarrow$ (i) follows by definition of $\bar{i}$ and $\mathcal{L}(i)$.

(i) $\Rightarrow$ (iii) By (i) there exist for each $n \in \mathbb{N}$ functions $g_{n}, h_{n} \in \mathcal{E}^{f i n}$ with

$$
\begin{gathered}
{ }^{\circ} i\left(h_{n}\right)-1 / n \leq i^{L}(f) \leq{ }^{\circ} i\left(g_{n}\right)+1 / n, \\
g_{n} \leq f \leq h_{n} .
\end{gathered}
$$

Put $\mathcal{H}_{n}:=\left\{e \in \mathcal{E}: g_{n} \leq e \leq h_{n}\right\}$. Then $\bigcap_{i=1}^{n} \mathcal{H}_{i} \neq \emptyset$ by (2). Hence there exists by saturation $e \in \bigcap_{i=1}^{\infty} \mathcal{H}_{i}$. This $e$ fulfills (iii). The remaining assertions are obvious.

1. Lemma. We have for $g, g_{1}, g_{2} \in{ }^{*} \mathbb{R}^{Y}$ and $0 \leq \alpha \in \mathbb{R}$

(i) $\underline{i}(g) \leq \bar{i}(g), \bar{i}(-g)=-\underline{i}(g)$;

(ii) $\overline{\bar{i}}(\alpha g)=\alpha \bar{i}(g), \underline{i}(\alpha g)=\alpha \underline{i}(g)$;

(iii) $g_{1} \leq g_{2} \Rightarrow \bar{i}\left(g_{1}\right) \leq \bar{i}\left(g_{2}\right), \underline{i}\left(g_{1}\right) \leq \underline{i}\left(g_{2}\right)$;

(iv) $\bar{i}\left(g_{1}+g_{2}\right) \leq \bar{i}\left(g_{1}\right)+\bar{i}\left(g_{2}\right), \underline{i}\left(g_{1}+g_{2}\right) \geq \underline{i}\left(g_{1}\right)+\underline{i}\left(g_{2}\right)$, whenever the right side is defined;

(v) $\underline{i}\left(g_{1}+g_{2}\right) \leq \underline{i}\left(g_{1}\right)+\bar{i}\left(g_{2}\right)$ if $0 \leq g_{1}, g_{2}$;

(vi) $i^{L}\left(g_{1}+g_{2}\right)=\underline{i}\left(g_{1}\right)+\bar{i}\left(g_{2}\right)$, if $0 \leq g_{1}, g_{2}$ and $g_{1}+g_{2} \in \mathcal{L}(i)$;

(vii) $0 \leq f_{n} \uparrow f, f_{n}, f \in \mathbb{R}^{Y} \Rightarrow \bar{i}\left(f_{n}\right) \uparrow \bar{i}(f)$.

Proof. (i)-(v) follow directly from the definitions of $\underline{i}$ and $\bar{i}$. (vi) follows by using (v), (iv) and (i).

(vii) According to (iii) there exists $\alpha \geq 0$ with $\bar{i}\left(f_{n}\right) \uparrow \alpha$. Let w.l.o.g. $\alpha<\infty$ and let $\varepsilon \in \mathbb{R}_{+}$be given. It suffices to show for each fixed $\delta \in \mathbb{R}_{+}$that

$$
\bar{i}(f) \leq(1+\delta)(\alpha+\varepsilon) .
$$

According to the definition of $\bar{i}$ there exist $g_{n} \in \mathcal{E}^{\text {fin }}$ with $f_{n} \leq g_{n}$ and ${ }^{\circ} i\left(g_{n}\right) \leq$ $\bar{i}\left(f_{n}\right)+\varepsilon / 2^{n}$. Then $h_{n}:=g_{1} \vee \ldots \vee g_{n}$ fulfill (use induction)

$$
f_{n} \leq h_{n} \in \mathcal{E}^{f i n},{ }^{\circ} i\left(h_{n}\right) \leq \bar{i}\left(f_{n}\right)+\sum_{i=1}^{n} \varepsilon / 2^{i} .
$$

Put $\mathcal{H}_{n}:=\left\{h \in \mathcal{E}: h_{n} \leq h, i(h) \leq \alpha+\varepsilon\right\}$. Then $\mathcal{H}_{n}$ are internal with $\emptyset \neq \mathcal{H}_{n} \downarrow$. Hence there exists $h \in \bigcap_{n=1}^{\infty} \mathcal{H}_{n}$. Then by (2) and $f_{n} \uparrow f$ we obtain $f \leq(1+\delta) h$. Hence we obtain (1).

Proof of Theorem 2. (i) follows directly from Lemma 1 and Theorem 1.

(ii) As $0 \leq f_{n}-f_{1} \uparrow f-f_{1} \in \mathbb{R}^{Y}$ we obtain the assertion from Lemma 1(vii) using (i).

(iii) follows from the definition of $\mathcal{L}(i)$. 
2. Lemma. Let $e \in \mathcal{E}^{\text {fin }}$ with $e(Y) \subset$ fin $\left({ }^{*} \mathbb{R}\right)$ be given. Then ${ }^{\circ} e \in \mathcal{L}(i)$. If furthermore $0 \leq e$, then $i^{L}\left({ }^{\circ} e\right) \leq{ }^{\circ} i(e)$.

Proof. Let w.l.o.g. $e \geq 0$ and put $e_{n}:=(e-1 / n)^{+}$. Then

$$
\begin{gathered}
0 \leq e_{n} \in \mathcal{E}^{f i n},{ }^{\circ} e_{n} \uparrow{ }^{\circ} e ; \\
\left|e_{n}-{ }^{\circ} e_{n}\right| \leq \alpha e \text { for all } 0<\alpha \in \mathbb{R} .
\end{gathered}
$$

By (2) we obtain $\bar{i}\left(\left|e_{n}-{ }^{\circ} e_{n}\right|\right)=0$. Hence ${ }^{\circ} e_{n} \in \mathcal{L}(i)$ by Theorem 1 . Since $\sup _{n \in \mathbb{N}} i^{L}\left({ }^{\circ} e_{n}\right) \leq{ }^{\circ} i(e)<\infty$ we obtain the assertions using (1) and Theorem 2(ii).

3. Lemma. Let $f_{0}: Y \rightarrow[0, \infty[$. Then

$$
\bar{i}\left(f_{0}\right)=\inf \left\{\lim i^{L}\left(f_{n}\right): f_{0} \leq \lim f_{n}, \mathcal{L}(i) \ni f_{n} \uparrow\right\}=: \tilde{i}^{L}\left(f_{0}\right) .
$$

Proof. If $\mathcal{L}(i) \ni f_{n} \uparrow$ and $f_{0} \leq \lim f_{n}$, then by Lemma 1 (vii):

$$
\bar{i}\left(f_{0}\right)=\bar{i}\left(\lim _{n \in \mathbb{N}}\left(f_{0} \wedge f_{n}\right)\right)=\lim _{n \in \mathbb{N}} \bar{i}\left(f_{0} \wedge f_{n}\right) \leq \lim _{n \in \mathbb{N}} \bar{i}\left(f_{n}\right) .
$$

Hence $\bar{i}\left(f_{0}\right) \leq \widetilde{i^{L}}\left(f_{0}\right)$. To prove the converse let $\bar{i}\left(f_{0}\right)<\infty$. Let $e \in \mathcal{E}^{f i n}$ with $f_{0} \leq e$. We have to prove

$$
\widetilde{i}^{L}\left(f_{0}\right) \leq{ }^{\circ} i(e) .
$$

Put $f_{n}:={ }^{\circ} e \wedge n={ }^{\circ}(e \wedge n)$. As $e \wedge n \in \mathcal{E}^{\text {fin }}$ we obtain $f_{n} \in \mathcal{L}(i)$ and $i^{L}\left(f_{n}\right) \leq$ ${ }^{\circ} i(e \wedge n) \leq{ }^{\circ} i(e)$ by Lemma 2 . Hence $\tilde{i}^{L}\left(f_{0}\right) \leq \lim _{n \rightarrow \infty} i^{L}\left(f_{n}\right) \leq{ }^{\circ} i(e)$, as $\mathcal{L}(i) \ni f_{n} \uparrow$ and $f_{0} \leq \lim f_{n}$.

The following concept is essentially due to Loeb (see [10, 4]).

4. Definition. We call $\langle J, j\rangle$ a complete integration structure if $J \subset \mathbb{R}^{Y}$ is a Stonian vector-lattice and $j: J \rightarrow \mathbb{R}$ is a positive linear functional with the following properties:

$$
\begin{gathered}
J \ni f_{n} \uparrow f \in \mathbb{R}^{Y} \text { and } \sup _{n \in \mathbb{N}} j\left(f_{n}\right)<\infty \Rightarrow f \in J \text { and } j\left(f_{n}\right) \uparrow j(f), \\
f, h \in J, \quad f \leq g \leq h \text { and } j(f)=j(h) \Rightarrow g \in J .
\end{gathered}
$$

According to Theorem $2,\left\langle\mathcal{L}(i), i^{L}\right\rangle$ is a complete integration structure. For a complete integration structure put, for $g \in \mathbb{R}^{Y}$,

- $\widetilde{j}(g):=\inf \left\{\lim j\left(f_{n}\right): g \leq \lim f_{n}, J \ni f_{n} \uparrow\right\}$,

- $j(g):=\sup \left\{\lim j\left(f_{n}\right): \lim f_{n} \leq g, J \ni f_{n} \downarrow\right\}$,

- $\mathcal{M}_{0}:=\left\{A \subset Y: 1_{A} \in J\right\}$,

- $\mathcal{M}:=\left\{A \subset Y: A \cap M_{0} \in \mathcal{M}_{0}\right.$ for all $\left.M_{0} \in \mathcal{M}_{0}\right\}$,

- $\widetilde{j}(A):=\widetilde{j}\left(1_{A}\right)$.

By Theorem 7.1 on page 103 of Floret [3] and by Proposition 10.7 and Corollaries 12.20 and 12.22 of Pfeffer [12] one obtains the following classical result.

5. Lemma. Let $\langle J, j\rangle$ be a complete integration structure. Then $J=\left\{f \in \mathbb{R}^{Y}\right.$ : $j(f)=\widetilde{j}(f) \in \mathbb{R}\}$ and

(i) $\tilde{j}$ is a complete and saturated measure on the $\sigma$-algebra $\mathcal{M}(\widetilde{j})$;

(ii) $\mathcal{M}(\widetilde{j})=\left\{A \subset Y:\left(\forall M_{0} \in \mathcal{M}_{0}\right) A \cap M_{0} \in \mathcal{M}_{0}\right\}$; 
(iii) $\mathcal{M}_{0}$ is a $\delta$-ring and $\mathcal{M}_{0}=\{A \in \mathcal{M}(\widetilde{j}): \widetilde{j}(A) \in \mathbb{R}\}$;

(iv) $\widetilde{j}(f)=\int f \widetilde{d j}$ for each $\mathcal{M}(\widetilde{j})$-measurable function $f: Y \rightarrow[0, \infty[$;

(v) $J=\left\{f \in \mathbb{R}^{Y}: f\right.$ is $\widetilde{j} \mid \mathcal{M}(\widetilde{j})$-Lebesgue-integrable $\}$ and $j(f)=\int f d \widetilde{j}$ for all $f \in J$;

(vi) $0 \leq f \in J$ and $0<r \Rightarrow\{f>r\} \in \mathcal{M}_{0}$.

Proof of Theorem 3. $\langle J, j\rangle:=\left\langle\mathcal{L}(i), i^{L}\right\rangle$ is a complete integration according to Theorem 2. Hence $\mathcal{L}(i)=\left\{f \in \mathbb{R}^{Y}:{\underset{\sim}{i}}^{L}(f)=\widetilde{i^{L}}(f) \in \mathbb{R}\right\}$ by Lemma 5 . By Lemma 3 we have $\widetilde{j}(f)=\widetilde{i^{L}}(f)=\bar{i}(f)$ for $f: Y \rightarrow\left[0, \infty\left[\right.\right.$, and hence $\widetilde{j}(A)=\widetilde{i^{L}}(A)=\bar{i}(A)$. Therefore we obtain (i)-(vi) of Theorem 3 from (i)-(vi) of Lemma 5.

6. Lemma. Let $e \in \mathcal{E}$ with $|e| \leq f \in \mathbb{R}^{Y}$ and $\underline{i}(f)<\infty$ be given. Then ${ }^{\circ} e \in \mathcal{L}(i)$ and $i^{L}\left({ }^{\circ} e\right)={ }^{\circ} i(e)$.

Proof. As $\underline{i}(|e|) \leq \underline{i}(f)<\infty$, it is easily seen that $i(|e|)$ is finite (otherwise $i\left(\frac{n|e|}{i(|e|)}\right)=$ $n$ and $\left.\frac{n|e|}{i(|e|)} \leq f\right)$. Hence ${ }^{\circ} e \in \mathcal{L}(i)$ by Lemma 2. Now let w.l.o.g. $e \geq 0$. As $i^{L}\left({ }^{\circ} e\right) \leq$ ${ }^{\circ} i(e)$ by Lemma 2 , it remains to prove ${ }^{\circ} i(e) \leq i^{L}\left({ }^{\circ} e\right)$. To this aim let $\varepsilon \in \mathbb{R}_{+}$be given. As $|e| \leq f \in \mathbb{R}^{Y}$ we obtain $\left|e-{ }^{\circ} e\right| \leq \varepsilon f$. Hence $\underline{i}\left(\left|e-{ }^{\circ} e\right|\right) \leq \varepsilon \underline{i}(f)$, whence $\underline{i}\left(\left|e-{ }^{\circ} e\right|\right)=0$. The assertion follows now, using Lemma $1(\mathrm{v})$, from

$$
{ }^{\circ} i(e)=\underline{i}(e) \leq \underline{i}\left(\left|e-{ }^{\circ} e\right|+{ }^{\circ} e\right) \leq \underline{i}\left(\left|e-{ }^{\circ} e\right|\right)+\bar{i}\left({ }^{\circ} e\right)=\bar{i}\left({ }^{\circ} e\right)=i^{L}\left({ }^{\circ} e\right) .
$$

7. Lemma. For $Y_{0} \subset Y$ we have

(i) $\bar{i}\left(Y_{0}\right)<\infty \Rightarrow i^{L}\left(M_{0}\right)=\bar{i}\left(Y_{0}\right)$ for some $\mathcal{M}_{0} \ni M_{0} \supset Y_{0}$;

(ii) $\underline{i}\left(Y_{0}\right)<\infty \Rightarrow i^{L}\left(M_{0}\right)=\underline{i}\left(Y_{0}\right)$ for some $\mathcal{M}_{0} \ni M_{0} \subset Y_{0}$;

(iii) $M_{0} \in \mathcal{M}_{0} \Rightarrow i^{L}\left(M_{0}\right)=\bar{i}\left(M_{0} \cap Y_{0}\right)+\underline{i}\left(M_{0} \backslash Y_{0}\right)$.

Proof. (i) By definition of $\bar{i}\left(Y_{0}\right)$ there exist

$$
e_{n} \in \mathcal{E}^{f i n}, 1_{Y_{0}} \leq e_{n} \leq 1, e_{n} \downarrow \text { with } \bar{i}\left(Y_{0}\right)=\lim _{n \rightarrow \infty}{ }^{\circ} i\left(e_{n}\right) .
$$

According to Lemma 2 and Theorem 2 we have ${ }^{\circ} e_{n} \in \mathcal{L}(i)$ and

$$
\bar{i}\left(Y_{0}\right) \geq \lim _{n \rightarrow \infty} i^{L}\left({ }^{\circ} e_{n}\right)=i^{L}\left(\lim _{n \rightarrow \infty}{ }^{\circ} e_{n}\right) .
$$

Put $M_{0}:=\left\{\lim _{n \rightarrow \infty}{ }^{\circ} e_{n}=1\right\}$. Then $Y_{0} \subset M_{0}$ according to $(1)$ and $\bar{i}\left(Y_{0}\right) \underset{(2)}{\geq} \bar{i}\left(\lim ^{\circ} e_{n}\right) \geq$ $\bar{i}\left(M_{0}\right)$, and hence $\bar{i}\left(Y_{0}\right)=\bar{i}\left(M_{0}\right)$. As $f:=\lim _{n \rightarrow \infty}{ }^{\circ} e_{n} \in \mathcal{L}(i)$ and $0 \leq f \leq 1$ we have $\{f=1\}=\bigcap_{n=2}^{\infty}\{f>1-1 / n\} \in \mathcal{M}_{0}$ by Theorem 3 (vi) and (iii). and

(ii) According to the definition of $\underline{i}\left(Y_{0}\right)$ there exist $\mathcal{E} \ni e_{n} \uparrow$ with $0 \leq e_{n} \leq 1_{Y_{0}}$

$$
\infty>\underline{i}\left(Y_{0}\right)=\lim _{n \rightarrow \infty}{ }^{\circ} i\left(e_{n}\right)=\lim _{n \rightarrow \infty} i^{L}\left({ }^{\circ} e_{n}\right)=i^{L}\left(\lim _{n \rightarrow \infty}{ }^{\circ} e_{n}\right),
$$

where the last two equalities follow from Lemma 6 and Theorem 2. Put $f_{0}:=$ $\lim _{n \rightarrow \infty}{ }^{\circ} e_{n}$ and $M_{0}:=\left\{f_{0}>0\right\}$. Then $M_{0} \subset Y_{0}$ and $f_{0} \in \mathcal{L}(i)$. According to Theorem 3 (vi) we obtain that $M_{n}:=\left\{f_{0}>1 / n\right\} \in \mathcal{M}_{0}$. As $M_{n} \uparrow M_{0}$ and $\sup i^{L}\left(1_{M_{n}}\right)=$ $\sup \underline{i}\left(M_{n}\right) \leq \underline{i}\left(Y_{0}\right)<\infty$, we obtain $M_{0} \in \mathcal{M}_{0}$ by Theorem 2 (ii). The assertion follows now from

$$
\underline{i}\left(Y_{0}\right)=i^{L}\left(f_{0}\right)=\bar{i}\left(f_{0}\right) \leq \bar{i}\left(M_{0}\right)=i^{L}\left(M_{0}\right) .
$$


(iii) Using Lemma 1 we obtain the assertion by

$$
\begin{aligned}
i^{L}\left(M_{0}\right) & =\underline{i}\left(1_{M_{0}}\right) \leq \underline{i}\left(1_{M_{0} \backslash Y_{0}}\right)+\bar{i}\left(1_{M_{0} \cap Y_{0}}\right)=\underline{i}\left(M_{0} \backslash Y_{0}\right)+\bar{i}\left(1_{M_{0}} \backslash 1_{M_{0} \backslash Y_{0}}\right) \\
& \leq \underline{i}\left(M_{0} \backslash Y_{0}\right)+\bar{i}\left(-1_{M_{0} \backslash Y_{0}}\right)+\bar{i}\left(M_{0}\right) \\
& =\underline{i}\left(M_{0} \backslash Y_{0}\right)+i^{L}\left(M_{0}\right)-\underline{i}\left(M_{0} \backslash Y_{0}\right) \\
& =i^{L}\left(M_{0}\right) .
\end{aligned}
$$

8. Lemma. Let $Y \supset Y_{n} \downarrow Y_{0}$ and $\underline{i}\left(Y_{1}\right)<\infty$. Then $\underline{i}\left(Y_{n}\right) \downarrow \underline{i}\left(Y_{0}\right)$.

Proof. Put $\alpha:=\lim _{n \rightarrow \infty} \underline{i}\left(Y_{n}\right)$. Let $\varepsilon \in \mathbb{R}_{+}$. We have to show

$$
\alpha-\varepsilon \leq \underline{i}\left(Y_{0}\right) .
$$

By definition of $\underline{i}$ there exist $0 \leq e_{n} \in \mathcal{E}^{\text {fin }}$ with $e_{n} \leq 1_{Y_{n}}$ and $\underline{i}\left(Y_{n}\right) \leq{ }^{\circ} i\left(e_{n}\right)+\varepsilon / 2^{n}$. Let $g_{n}:=e_{1} \wedge \ldots \wedge e_{n}$. Then

$$
\begin{gathered}
0 \leq g_{n} \in \mathcal{E}^{f i n}, \quad g_{n} \leq 1_{Y_{n}}, \quad g_{n} \downarrow, \\
\underline{i}\left(Y_{n}\right) \leq{ }^{\circ} i\left(g_{n}\right)+\sum_{i=1}^{n} \varepsilon / 2^{i} \text { (which follows by induction). }
\end{gathered}
$$

Put $\mathcal{C}_{n}:=\left\{e \in \mathcal{E}: 0 \leq e \leq g_{n}\right.$ and $\left.i(e) \geq \alpha-\varepsilon\right\}$. Then $\emptyset \neq \mathcal{C}_{n} \downarrow$. By saturation there exists $e \in \bigcap_{n=1}^{\infty} \mathcal{C}_{n}$. Hence $0 \leq e \in \mathcal{E}^{f i n}, i(e) \geq \alpha-\varepsilon$ and $e \leq g_{n}$. Therefore $e \leq 1_{Y_{0}}$ and hence (1) follows.

9. Lemma. Let $\mathcal{C}$ be a system of internal sets with $\underline{i}(C)=\infty$ for all $C \in \mathcal{C}$. Assume that $\mathcal{C}$ is downwards directed, has the finite intersection property and fulfills $|\mathcal{C}| \leq|\widehat{S}|$. Then $\underline{i}\left(\bigcap_{C \in \mathcal{C}} C\right)=\infty$.

Proof. Put $\mathcal{S}_{C}^{n}:=\left\{e \in \mathcal{E}: 0 \leq e \leq 1_{C}, i(e) \geq n\right\}$. Then $\left\{\mathcal{S}_{C}^{n}: C \in \mathcal{C}, n \in \mathbb{N}\right\}$ is a system of internal sets with finite intersection and cardinality $\leq|\widehat{S}|$. Hence $\bigcap_{n \in \mathbb{N}, C \in \mathcal{C}} \mathcal{S}_{C}^{n} \neq \emptyset$. Let $e \in \bigcap_{n \in \mathbb{N}, C \in \mathcal{C}} \mathcal{S}_{C}^{n}$. Then $e \in \mathcal{E}$ and $e \leq 1_{\bigcap_{C \in \mathcal{C}} C}$ with $i(e) \geq n$ for all $n \in \mathbb{N}$. Hence for each $n \in \mathbb{N}$ there exists $e \in \mathcal{E}^{f i n}$ with $i(|e|) \geq n$ and $e \leq 1_{\bigcap_{C \in \mathcal{C}} C}$. Hence $\underline{i}\left(\bigcap_{C \in \mathcal{C}} C\right)=\infty$.

10. Lemma. Let $Y_{0} \subset Y$ with $\underline{i}\left(Y_{0}\right)=\infty$ and $c_{0}:=\sup \left\{\underline{i}(T): T \subset Y_{0}, \underline{i}(T)<\right.$ $\infty\}<\infty$. Then there exists an internal set $C \subset Y_{0}$ with $\underline{i}(C)=\infty$.

Proof. As $\underline{i}\left(Y_{0}\right)=\infty$, there exist $0 \leq e_{n} \in \mathcal{E}^{\text {fin }}$ with $e_{n} \leq 1_{Y_{0}}$ and ${ }^{\circ} i\left(e_{n}\right) \underset{n \rightarrow \infty}{\rightarrow} \infty$. Choose $n_{0}$ with $c_{0}<{ }^{\circ} i\left(e_{n_{0}}\right)$ and put $C:=\left\{e_{n_{0}}>0\right\}$. Then $C$ is an internal subset of $Y_{0}$ and $c_{0}<\underline{i}\left(e_{n_{0}}\right) \leq \underline{i}(C)$. Hence $\underline{i}(C)=\infty$ by definition of $c_{0}$.

11. Lemma. Let $0 \leq f \in \mathbb{R}^{Y}$ and $\underline{i}(\{f \neq 0\})$. Then $\underline{i}(f)=0$.

Proof. Let $0 \leq e \leq f$ with $e \in \mathcal{E}^{f i n}$ be given. Then as $e$ is internal there exists $c \in$ $\mathbb{R}_{+}$with $e \leq c$. Hence $e \leq c 1_{\{f \neq 0\}}$ and therefore ${ }^{\circ} i(e)=\underline{i}(e) \leq \underline{i}\left(c 1_{\{f \neq 0\}}\right)=0$.

12. Lemma. Let $B \in \mathcal{M}(\bar{i})$. Then $B \in \mathcal{M}(\underline{i})$ if the following condition is fulfilled: $A$ internal, $\underline{i}(A)=\infty$ and $\sup \{\underline{i}(T): T \subset A, \underline{i}(T)<\infty\}<\infty$

$$
\Rightarrow \underline{i}(A \cap B)=\infty \text { or } \underline{i}(A \backslash B)=\infty \text {. }
$$


Proof. We have to show that for $A \subset Y$

$$
\underline{i}(A) \leq \underline{i}(A \cap B)+\underline{i}(A \backslash B) .
$$

If $\underline{i}(A)<\infty$, then by Lemma 7 (ii) there exists $M_{0} \in \mathcal{M}_{0}$ with $M_{0} \subset A$ and $\underline{i}(A)=i^{L}\left(M_{0}\right)$. Then $M_{0} \cap B, M_{0} \cap \bar{B} \in \mathcal{M}_{0}$ by Theorem 3 (ii) and hence

$$
\underline{i}(A)=i^{L}\left(M_{0}\right)=i^{L}\left(M_{0} \cap B\right)+i^{L}\left(M_{0} \backslash B\right) \leq \underline{i}(A \cap B)+\underline{i}(A \backslash B) .
$$

If $\infty=\underline{i}(A)=\sup \{\underline{i}(T): T \subset A, \underline{i}(T)<\infty\}$, then we obtain as just proved

$$
\underline{i}(T) \leq \underline{i}(T \cap B)+\underline{i}(T \backslash B)
$$

for all $T$ with $\underline{i}(T)<\infty$. Hence (1) follows. Now consider the remaining case, namely $\underline{i}(A)=\infty$ and $\sup \{\underline{i}(T): T \subset A, \underline{i}(T)<\infty\}<\infty$. Then by Lemma 10 there exists an internal set $A^{\prime}$ with $A^{\prime} \subset A$ and $\underline{i}\left(A^{\prime}\right)=\infty$. Hence we obtain $\underline{i}\left(A^{\prime} \cap B\right)=\infty$ or $\underline{i}\left(A^{\prime} \backslash B\right)=\infty$ by assumption. As $A^{\prime} \subset A$, this implies (1).

Let us remark that Lemma 12 yields a condition for $\mathcal{M}(\underline{i})=\mathcal{M}(\bar{i})$.

Proof of Theorem 4. (ii) It suffices to show $A \notin \mathcal{M}(\bar{i}) \Rightarrow A \notin \mathcal{M}(\underline{i})$.

As $A \notin \mathcal{M}(\bar{i})$, we obtain by Theorem 3 (ii) that there exists $M_{0} \in \mathcal{M}_{0}$ with $A \cap M_{0} \notin \mathcal{M}_{0}$. Now $\bar{i}\left(A \cap M_{0}\right) \leq \bar{i}\left(M_{0}\right)<\infty$ and hence $\underline{i}\left(A \cap M_{0}\right)<\bar{i}\left(A \cap M_{0}\right)$ by definition of $\mathcal{M}_{0}$. Using Lemma 7 (iii) we obtain

$$
\underline{i}\left(M_{0}\right)=\bar{i}\left(M_{0} \cap A\right)+\underline{i}\left(M_{0} \backslash A\right)>\underline{i}\left(M_{0} \cap A\right)+\underline{i}\left(M_{0} \backslash A\right) ;
$$

hence $A \notin \mathcal{M}(\underline{i})$.

(iii) Let $A \in \mathcal{M}_{0}$. We have to prove (see Lemma 1 (iv)) that

$$
\underline{i}(B) \leq \underline{i}(B \cap A)+\underline{i}(B \backslash A) \text { for each } B \subset Y .
$$

If $\underline{i}(B)<\infty$, then by Lemma 7 (ii) there exists $M_{0} \in \mathcal{M}_{0}$ with $M_{0} \subset B$ and $i^{L}\left(M_{0}\right)=\underline{i}(B)$. Hence

$$
\underline{i}(B)=i^{L}\left(M_{0}\right)=i^{L}\left(M_{0} \cap A\right)+i^{L}\left(M_{0} \backslash A\right) \leq \underline{i}(B \cap A)+\underline{i}(B \backslash A),
$$

i.e. (1) holds. Let $\underline{i}(B)=\infty$. According to Lemma 1 (v) we obtain $\underline{i}(B) \leq$ $\bar{i}(B \cap A)+\underline{i}(B \backslash A) \leq i^{L}(A)+\underline{i}(B \backslash A)$; hence $\underline{i}(B \backslash A)=\infty$ and (1) holds.

(i) As $\mathcal{M}(\underline{i})$ is an algebra, to prove that $\mathcal{M}(\underline{i})$ is a $\sigma$-algebra, it is sufficient to show

$$
A_{n} \in \mathcal{M}(\underline{i}) \wedge A_{n} \downarrow A \Rightarrow A \in \mathcal{M}(\underline{i}) .
$$

As $A \in \mathcal{M}(\bar{i})$ by (ii), it suffices to prove according to Lemma 12

$$
\begin{gathered}
B \text { internal, } \underline{i}(B)=\infty \text { and } \sup \{\underline{i}(T): T \subset B, \underline{i}(T)<\infty\}<\infty \\
\Rightarrow \underline{i}(B \cap A)=\infty \text { or } \underline{i}(B \backslash A)=\infty .
\end{gathered}
$$

Ad (3): Let w.l.o.g. $\underline{i}(B \backslash A)<\infty$. We inductively construct internal sets $C_{n}$ with

$$
C_{n} \subset B \cap A_{n}, C_{n} \downarrow \text { and } \underline{i}\left(C_{n}\right)=\infty .
$$

Then $\underline{i}\left(\bigcap_{n=1}^{\infty} C_{n}\right)=\infty$ by Lemma 9 and hence $\underline{i}(B \cap A) \geq \underline{i}\left(\bigcap_{n=1}^{\infty} C_{n}\right)=\infty$, whence (3) holds.

As $\underline{i}\left(B \backslash A_{n}\right) \leq \underline{i}(B \backslash A)<\infty$, we obtain from $A_{n} \in \mathcal{M}(\underline{i})$

$$
\underline{i}\left(B \cap A_{n}\right)=\infty \text { for } n \in \mathbb{N} .
$$

According to Lemma 10 applied to $Y_{0}:=B \cap A_{1}$, there exists an internal set $C_{1} \subset B \cap A_{1}$ with $\underline{i}\left(C_{1}\right)=\infty$. Assume inductively that $C_{1}, \ldots, C_{n}$ are constructed 
with $C_{1} \supset \ldots \supset C_{n}, C_{i} \subset B \cap A_{i}$ and $\underline{i}\left(C_{i}\right)=\infty$ for $i=1, \ldots, n$. As $A_{n+1} \in \mathcal{M}(\underline{i})$, we obtain

$$
\infty=\underline{i}\left(C_{n}\right)=\underline{i}\left(C_{n} \cap A_{n+1}\right)+\underline{i}\left(C_{n} \backslash A_{n+1}\right),
$$

which implies $\underline{i}\left(C_{n} \cap A_{n+1}\right)=\infty$ as $\underline{i}\left(C_{n} \backslash A_{n+1}\right) \leq \underline{i}\left(B \backslash A_{n+1}\right) \leq \underline{i}(B \backslash A)<\infty$.

According to Lemma 10 applied to $Y_{0}:=C_{n} \cap A_{n+1}$ we obtain an internal set $C_{n+1} \subset C_{n} \cap A_{n+1}$ with $\underline{i}\left(C_{n+1}\right)=\infty$. Hence (4) is proven by induction.

Now we prove that $\underline{i}$ is a measure on $\mathcal{M}(\underline{i})$. As $\underline{i}$ is additive on $\mathcal{M}(\underline{i})$, it suffices to show that

$$
\mathcal{M}(\underline{i}) \ni A_{n} \uparrow A \Rightarrow \underline{i}\left(A_{n}\right) \rightarrow \underline{i}(A) .
$$

If $\underline{i}(A)<\infty$, then $\underline{i}\left(A \backslash A_{n}\right) \rightarrow 0$ by Lemma 8 , and hence (6) holds.

Now let $\underline{i}(A)=\infty$ and assume indirectly that $\sup \underline{i}\left(A_{n}\right)=: c_{1}<\infty$. First, let $\sup \{\underline{i}(T): T \subset A, \underline{i}(T)<\infty\}=\infty$. According to Lemma 7 (ii) there exists $M_{0} \subset A$ with $i^{L}\left(M_{0}\right)>c_{1}$. As $A_{n} \in \mathcal{M}(\bar{i})$ by (ii), we obtain $M_{0} \cap A_{n} \in \mathcal{M}_{0}$ by Theorem 3 (ii). Hence we obtain the following contradiction:

$$
c_{1}<i^{L}\left(M_{0}\right)=\lim _{n \rightarrow \infty} i^{L}\left(M_{0} \cap A_{n}\right)=\lim _{n \rightarrow \infty} \underline{i}\left(M_{0} \cap A_{n}\right) \leq \lim \underline{i}\left(A_{n}\right) \leq c_{1} .
$$

Now let $\sup \{\underline{i}(T): T \subset A, \underline{i}(T)<\infty\}<\infty$. We construct internal sets $C_{n} \subset$ $A \backslash A_{n}$ with $C_{n} \downarrow$ and $\underline{i}\left(C_{n}\right)=\infty$. This yields a contradiction since $\bigcap_{n=1}^{\infty} C_{n} \subset$ $\bigcap_{n=1}^{\infty}\left(A \backslash A_{n}\right)=\emptyset$ implies $C_{n}=\emptyset$ for some $n$.

As $\underline{i}\left(A \backslash A_{1}\right)=\infty$, we obtain by Lemma 10 an internal set $C_{1} \subset A \backslash A_{1}$ with $\underline{i}\left(C_{1}\right)=\infty$. Now assume inductively that internal sets $C_{n} \subset C_{n-1} \subset \ldots \subset C_{1}$ with $C_{j} \subset A \backslash A_{j}$ and $\underline{i}\left(C_{j}\right)=\infty$ for $j=1, \ldots, n$ are constructed. As $A \backslash A_{n+1} \in \mathcal{M}(\underline{i})$, we have

$$
\infty=\underline{i}\left(C_{n}\right)=\underline{i}\left(C_{n} \cap\left(A \backslash A_{n+1}\right)\right)+\underline{i}\left(C_{n} \backslash\left(A \backslash A_{n+1}\right)\right) .
$$

Since $\underline{i}\left(C_{n} \backslash\left(A \backslash A_{n+1}\right)\right) \leq \underline{i}\left(A_{n+1}\right)<\infty$, we obtain $\underline{i}\left(C_{n} \cap\left(A \backslash A_{n+1}\right)\right)=\infty$. Hence by Lemma 10 there exists an internal set $C_{n+1} \subset C_{n} \cap\left(A \backslash A_{n+1}\right)$ with $\underline{i}\left(C_{n+1}\right)=\infty$. Hence we have proven that $\underline{i}$ is a measure on $\mathcal{M}(\underline{i})$. The completeness of $\underline{i} \mid \mathcal{M}(\underline{i})$ follows from the monotonicity of $\underline{i}$ and the definition of $\mathcal{M}(\underline{i})$.

(v) Now let $0 \leq f \in \mathcal{L}(i)$. Then according to Theorem 3 (vi) and (iii) we have for each $r>0$

$$
\{f>r\} \in \mathcal{M}_{0} \subset \mathcal{M}(\underline{i}) \text {. }
$$

Hence $f$ is $\mathcal{M}(\underline{i})$-measurable. It suffices to prove $i^{L}(f)=\int f d \underline{i}$. According to $(7)$ there exist $f_{n}=\sum_{j=1}^{k_{n}} \alpha_{j, n} 1_{M_{j, n}}$ with $M_{j, n} \in \mathcal{M}_{0}$ and $f_{n} \uparrow f$. Hence according to Theorem 2 and the properties of the Lebesgue-integral

$$
i^{L}(f)=\lim i^{L}\left(f_{n}\right)=\lim \sum_{j=1}^{k_{n}} \alpha_{j, n} \underline{i}\left(M_{j, n}\right)=\lim \int f_{n} d \underline{i}=\int f d \underline{i} .
$$

To prove (iv) we show at first that for each $\underline{i} \mid \mathcal{M}(\underline{i})$-Lebesgue-integrable function $f \geq 0$ there exist $0 \leq f_{0} \in \mathcal{L}(i)$ with

$$
f_{0}=f \underline{i} \mid \mathcal{M}(\underline{i}) \text {-a.e. and } i^{L}\left(f_{0}\right)=\int f d \underline{i} .
$$

Choose $\mathcal{M}(\underline{i})$-elementary functions $f_{n}$ with $0 \leq f_{n} \uparrow f$. Using Lemma 7 (ii) one can construct $g_{n} \in \mathcal{L}(i)$ with $0 \leq g_{n} \leq f_{n}, g_{n} \uparrow$ and $g_{n}=f_{n} \underline{i} \mid \mathcal{M}(\underline{i})$-a.e. Put $f_{0}:=\lim g_{n} \geq 0$. Using $(\mathrm{v})$ and Theorem 2 it is easy to see that $0 \leq f_{0} \in \mathcal{L}(i)$ fulfills (8). 
(iv) Let $0 \leq f$ be $\mathcal{M}(\underline{i})$-measurable. We first prove

$$
\underline{i}(f) \geq \int f d \underline{i} .
$$

As $0 \leq f$ is $\mathcal{M}(\underline{i})$-measurable, there exist $\mathcal{M}(\underline{i})$-elementary functions

$$
f_{n}=\sum_{j=1}^{k_{n}} \alpha_{j, n} 1_{M_{j, n}} \uparrow f .
$$

Hence by Lemma 1 we obtain

$$
\underline{i}(f) \geq \underline{i}\left(f_{n}\right) \geq \sum_{j=1}^{k_{n}} \underline{i}\left(\alpha_{j, n} 1_{M_{j, n}}\right)=\sum_{j=1}^{k_{n}} \alpha_{j, n} \underline{i}\left(M_{j, n}\right)=\int f_{n} d \underline{i} \uparrow \int f d \underline{i},
$$

which shows (9). Because of (9) it suffices to show

$$
\underline{i}(f) \leq \int f d \underline{i} \text { for } \int f d \underline{i}<\infty .
$$

According to (8) there exists $0 \leq f_{0} \in \mathcal{L}(i)$ with

$$
f_{0}=f \underline{i} \mid \mathcal{M}(\underline{i}) \text {-a.e. and } i^{L}\left(f_{0}\right)=\int f d \underline{i} .
$$

To prove (10) it therefore suffices to show

$$
\underline{i}(f) \leq i^{L}\left(f_{0}\right) .
$$

We obtain from Lemma $1(\mathrm{v})$

$$
\underline{i}(f) \leq \underline{i}\left(\left|f-f_{0}\right|\right)+\bar{i}\left(f_{0}\right)=\underline{i}\left(\left|f-f_{0}\right|\right)+i^{L}\left(f_{0}\right) .
$$

As $\underline{i}\left(\left|f-f_{0}\right|\right)=0$ by Lemma 11, we obtain (12).

Proof of Example 5. (i) follows from the monotonicity of $\nu$ on $\mathcal{R}$ and the definition of $\underline{\nu}$ and $\bar{\nu}$.

(ii) We first show $\underline{\nu}(A) \leq \underline{i_{\nu}}(A)$. To prove this consider the following two cases:

$$
\nu(R) \text { is finite for all } R \in \mathcal{R} \text { with } R \subset A \text {; }
$$

$$
\text { there exists } R_{0} \in \mathcal{R} \text { with } R_{0} \subset A \text { and } \nu\left(R_{0}\right) \text { infinite. }
$$

Ad (1): Let $R \in \mathcal{R}, R \subset A$ be given. Then $\mathcal{E}^{\text {fin }} \ni e:=1_{R} \leq 1_{A}$ and hence ${ }^{\circ} \nu(R)={ }^{\circ} i_{\nu}(e) \leq \underline{i_{\nu}}\left(1_{A}\right)$. Whence $\underline{\nu}(A) \leq \underline{i_{\nu}}\left(1_{A}\right)=\underline{i_{\nu}}(A)$.

Ad (2): Let $\alpha \in \mathbb{R}_{+}$and put $e:=\frac{\alpha}{\nu\left(R_{0}\right)} 1_{R_{0}}$. Then $e \in \mathcal{E}^{f i n}, i_{\nu}(e)=\alpha$ and $e \leq 1_{A}$. Hence $\underline{i_{\nu}}\left(1_{A}\right) \geq{ }^{\circ} i_{\nu}(e)=\alpha$. As $\alpha \in \mathbb{R}_{+}$is arbitrary, we obtain $\underline{i_{\nu}}(A)=\infty \geq \underline{\nu}(A)$. It remains to prove $i_{\nu}(A) \leq \underline{\nu}(A)$. Let $e \in \mathcal{E}^{\text {fin }}$ with $0 \leq e \leq 1_{A}$ be given. We have $e=\sum_{j=1}^{n} \alpha_{j} 1_{R_{j}}$ with disjoint $\emptyset \neq R_{j} \in \mathcal{R}$. Then $R:=\bigcup_{\alpha_{j}>0} R_{j} \in \mathcal{R}$ and $R \subset A$. Furthermore $e \leq 1_{R}$ and hence

$$
{ }^{\circ} i_{\nu}(e) \leq{ }^{\circ} i_{\nu}\left(1_{R}\right) \underset{(i)}{=} \underline{\nu}(R) \leq \underline{\nu}(A) .
$$

Hence $\underline{i_{\nu}}(A)=\underline{i_{\nu}}\left(1_{A}\right) \leq \underline{\nu}(A)$. This proves $\underline{\nu}(A)=\underline{i_{\nu}}(A)$.

The proof for $\bar{\nu}(A)=\overline{i_{\nu}}(A)$ runs similarly. (ii).

(iii) As $\mathcal{M}_{0}=\left\{A \subset Y: \underline{i_{\nu}}\left(1_{A}\right)=\bar{i}_{\nu}\left(1_{A}\right)<\infty\right\}$ by definition, we obtain (iii) by

(iv) As $\underline{\nu}(A)=\underline{i_{\nu}}(A), \bar{\nu}(A)=\overline{i_{\nu}}(A)$ for each $A \subset Y$, we obtain $\mathcal{M}(\underline{\nu}) \subset \mathcal{M}(\bar{\nu})$ by Theorem 4 (ii). Let $R \in \mathcal{R}$; it remains to show $R \in \mathcal{M}(\underline{\nu})$. Let $A \subset Y$. As $\nu$ is a 
content on $\mathcal{R}$ we obtain from the definition of $\underline{\nu}$ that $\underline{\nu}(A) \leq \underline{\nu}(A \cap R)+\underline{\nu}(A \backslash R)$. Hence $R \in \mathcal{M}(\underline{\nu})$.

(v) follows from Theorem 4 and Theorem 3 using (ii).

13. Lemma. Let $0 \leq e \in \mathcal{E}$ and let $0 \leq r \in \mathbb{R}$. Then there exists $e_{r} \in \mathcal{E}$ with $1_{\left\{{ }^{0} e>r\right\}} \leq e_{r} \leq 1_{\left\{{ }^{\circ} e \geq r\right\}}$.

Proof. Put $e_{r}=1 \wedge n(e-e \wedge r)$ with $n \in{ }^{*} \mathbb{N} \backslash \mathbb{N}$. Then $e_{r} \in \mathcal{E}$ and $e_{r}$ fulfills the above inequalities.

14. Lemma. Let $B \subset Y$ be internal with $\underline{i}(B)=\infty$. Then for every $r \in[0,1]$ and $e \in \mathcal{E}$ with $0 \leq e$ we have

$$
\underline{i}\left(B \cap\left\{{ }^{\circ} e \leq r\right\}\right)=\infty \text { or } \underline{i}\left(B \cap\left\{{ }^{\circ} e \geq r\right\}\right)=\infty .
$$

Proof. As $\underline{i}(B)=\infty$, there exists $g_{n} \in \mathcal{E}^{\text {fin }}$ with

$$
0 \leq g_{n} \leq 1_{B}, \quad{ }^{\circ} i\left(g_{n}\right) \uparrow \infty .
$$

By Lemma 13 there exists $e_{r} \in \mathcal{E}$ with

$$
1_{\left\{{ }^{\circ} e>r\right\}} \leq e_{r} \leq 1_{\left\{{ }^{\circ} e \geq r\right\}} .
$$

From (1) and (2) we obtain

$$
\begin{gathered}
g_{n} \wedge e_{r} \in \mathcal{E}^{f i n}, g_{n} \wedge e_{r} \leq 1_{B \cap\left\{{ }^{\circ} e \geq r\right\}}, \\
\mathcal{E}^{f i n} \ni g_{n}-g_{n} \wedge e_{r} \leq 1_{B \cap\left\{{ }^{\circ} e \leq r\right\}} .
\end{gathered}
$$

As ${ }^{\circ} i\left(g_{n}\right)={ }^{\circ} i\left(g_{n} \wedge e_{r}\right)+{ }^{\circ} i\left(g_{n}-g_{n} \wedge e_{r}\right)$ and ${ }^{\circ} i\left(g_{n}\right) \uparrow \infty$, there exists a subsequence with ${ }^{\circ} i\left(g_{n} \wedge e_{r}\right) \rightarrow \infty$ or ${ }^{\circ} i\left(g_{n}-g_{n} \wedge e_{r}\right) \rightarrow \infty$. Whence $\underline{i}\left(B \cap\left\{{ }^{\circ} e \leq r\right\}\right)=\infty$ or $\underline{i}\left(B \cap\left\{{ }^{\circ} e \geq r\right\}\right)=\infty$.

15. Lemma. (i) $e \in \mathcal{E}^{\text {fin }} \Rightarrow{ }^{\circ} e$ is $\sigma\left(\mathcal{M}_{0}\right)$-measurable.

(ii) $e \in \mathcal{E} \Rightarrow{ }^{\circ}$ e is $\mathcal{M}(\underline{i})$-measurable.

Proof. Using that $\mathcal{E}$ is a Stonian lattice we may assume in (i) and (ii) that $0 \leq e \leq 1$.

(i) According to Lemma 2 we have ${ }^{\circ} e \in \mathcal{L}(i)$. The assertion follows now from Theorem 3 (vi).

(ii) We first show that ${ }^{\circ} e$ is $\mathcal{M}(\bar{i})$-measurable. Let $M_{0} \in \mathcal{M}_{0}$ be given. We prove

$$
1_{M_{0}} \wedge^{\circ} e \in \mathcal{L}(i) .
$$

Let $\varepsilon \in \mathbb{R}_{+}$be given. There exists $g$ with

$$
g \in \mathcal{E}^{f i n}, 1_{M_{0}} \leq g \leq 1 \text { and } i^{L}\left({ }^{\circ} g\right) \leq{ }^{\circ} i(g) \leq i^{L}\left(M_{0}\right)+\varepsilon .
$$

As $^{\circ}(e \wedge g) \leq 1_{M_{0}} \wedge{ }^{\circ} e+{ }^{\circ} g-1_{M_{0}}$, we obtain from Lemma $1(\mathrm{v})$

$$
\underline{i}\left({ }^{\circ}(e \wedge g)\right) \leq \underline{i}\left(1_{M_{0}} \wedge{ }^{\circ} e\right)+\bar{i}\left({ }^{\circ} g-1_{M_{0}}\right) .
$$

As ${ }^{\circ}(e \wedge g),{ }^{\circ} g \in \mathcal{L}(i)$ by Lemma (2), we obtain from (2)

$$
\bar{i}\left(1_{M_{0}} \wedge^{\circ} e\right) \leq \bar{i}\left({ }^{\circ}(e \wedge g)\right) \leq \underline{i}\left(1_{M_{0}} \wedge^{\circ} e\right)+\varepsilon .
$$

This proves (1). Now let $r>0$. Then by Theorem 3 (vi) we have $\left\{{ }^{\circ} e>r\right\} \cap M_{0}=$ $\left\{1_{M_{0}} \wedge^{\circ} e>r\right\} \in \mathcal{M}_{0}$. Hence we obtain by Theorem 3 (ii) that

$$
{ }^{\circ} e \text { is } \mathcal{M}(\bar{i}) \text {-measurable. }
$$


Now let $0 \leq r \in \mathbb{R}$ be given and put $B_{r}:=\left\{{ }^{\circ} e \geq r\right\}$. Then $B_{r} \in \mathcal{M}(\bar{i})$ according to (3). To prove $B_{r} \in \mathcal{M}(\underline{i})$ we have to show according to Lemma 12:

$$
\left\{\begin{array}{c}
A \text { internal, } \underline{i}(A)=\infty \text { and } \sup \{\underline{i}(T): T \subset A, \underline{i}(T)<\infty\}<\infty \\
\Rightarrow \underline{i}\left(A \cap B_{r}\right)=\infty \text { or } \underline{i}\left(A \backslash B_{r}\right)=\infty .
\end{array}\right.
$$

Define $r_{0}:=\sup \left\{s \in[0,1]: \underline{i}\left(A \cap B_{s}\right)=\infty\right\} \in[0,1]$. If $r<r_{0}$, then $\underline{i}\left(A \cap B_{r}\right)=$ $\infty$. If $r>r_{0}$, then $\underline{i}\left(A \cap B_{r}\right)<\infty$ and hence using Lemma 14: $\underline{i}\left(A \backslash B_{r}\right)=$ $\underline{i}\left(A \cap\left\{{ }^{\circ} e<r\right\}\right)=\infty$. This proves (3) for $r \neq r_{0}$. It remains to prove (3) if $r=r_{0}$. Let $T_{n}:=A \cap\left\{e>r_{0}-1 / n\right\}$. Then $T_{n}$ are internal with $T_{n} \downarrow$ and $\underline{i}\left(T_{n}\right)=\infty$ by definition of $r_{0}$. Hence $\underline{i}\left(\bigcap_{n=1}^{\infty} T_{n}\right)=\infty$ by Lemma 9 . As $\bigcap_{n=1}^{\infty} T_{n} \subset A \cap\left\{{ }^{\circ} e \geq r_{0}\right\}$, we obtain $\underline{i}\left(A \cap B_{r_{0}}\right)=\infty$.

16. Lemma. If $0 \leq e \in \mathcal{E}$, then

(i) $\underline{i}(e)=\bar{i}(e)={ }^{\circ} i(e)$;

(ii) $\underline{i}\left({ }^{\circ} e\right)=\bar{i}\left({ }^{\circ} e\right)$.

Proof. (i) If $e \in \mathcal{E}^{\text {fin }}$, then the assertion follows by definition of $\bar{i}$ and $\underline{i}$. If $e \notin \mathcal{E}^{\text {fin }}$, then $i(e)$ is positive infinite. It suffices to prove $\underline{i}(e)=\infty$. Let $\alpha \in \mathbb{R}_{+}$and put $e_{\alpha}=\frac{\alpha}{i(e)} e$. Then $i\left(e_{\alpha}\right)=\alpha, e_{\alpha} \in \mathcal{E}^{f i n}$ and $e_{\alpha} \leq e$. Hence $\underline{i}(e) \geq \alpha$ for all $\alpha \in \mathbb{R}_{+}$.

(ii) ${ }^{\circ} e$ is $\mathcal{M}(\underline{i})$-measurable by Lemma 15 . As

$$
\begin{aligned}
& \bar{i}\left({ }^{\circ} e\right)=\int{ }^{\circ} e d \bar{i}=\lim _{n \rightarrow \infty} \int\left({ }^{\circ} e \wedge n\right) d \bar{i}=\lim _{n \rightarrow \infty} \bar{i}\left({ }^{\circ} e \wedge n\right), \\
& \underline{i}\left({ }^{\circ} e\right)=\int{ }^{\circ} e d \underline{i}=\lim _{n \rightarrow \infty} \int\left({ }^{\circ} e \wedge n\right) d \underline{i}=\lim _{n \rightarrow \infty} \underline{i}\left({ }^{\circ} e \wedge n\right),
\end{aligned}
$$

we may assume that $0 \leq e \leq 1$. According to Theorem 4 (iv) it suffices to show that ${ }^{\circ} e \in \mathcal{L}(i)$ if ${ }^{\circ} e$ is $\underline{i} \mid \mathcal{M}(\underline{i})$-Lebesgue-integrable. Therefore let ${ }^{\circ} e$ be $\underline{i} \mid \mathcal{M}(\underline{i})$-Lebesgueintegrable. We show $\left\{{ }^{\circ} e>r\right\} \in \mathcal{M}_{0}$ for each $r>0$; by Lemma 13 there exists $0 \leq e_{r} \in \mathcal{E}$ with $1_{\left\{{ }^{\circ} e>r\right\}} \leq e_{r} \leq 1_{\left\{{ }^{\circ} e \geq r\right\}}$. Hence by (i)

$$
\bar{i}\left(\left\{{ }^{\circ} e>r\right\}\right) \leq \bar{i}\left(e_{r}\right)=\underline{i}\left(e_{r}\right) \leq \underline{i}\left\{^{\circ} e \geq r\right\}<\infty .
$$

As $\left\{{ }^{\circ} e>r\right\} \in \mathcal{M}(\bar{i})$ by Lemma 15 (ii), we obtain that $\left\{{ }^{\circ} e>r\right\} \in \mathcal{M}_{0}$ by Theorem 3 (iii). As ${ }^{\circ} e$ is $\mathcal{M}(\bar{i})$-measurable, we obtain therefore for $k, n \in \mathbb{N}$ that $\left\{k / 2^{n} \leq{ }^{\circ} e<\right.$ $\left.(k+1) / 2^{n}\right\} \in \mathcal{M}_{0}$ (use Theorem 3 (iii)). Hence $f_{n}:=\sum_{k=1}^{n 2^{n}} \frac{k}{2^{n}} 1_{\left\{\frac{k}{2^{n}} \leq^{\circ} e<\frac{k+1}{2^{n}}\right\}} \in \mathcal{L}(i)$ and $f_{n} \uparrow^{\circ} e$. By Theorem 4 (v) we obtain

$$
\sup i^{L}\left(f_{n}\right)=\sup \int f_{n} d \underline{i} \leq \int{ }^{\circ} e d \underline{i}<\infty .
$$

Hence Theorem 2 (ii) implies ${ }^{\circ} e \in \mathcal{L}(i)$

Proof of Theorem 6. We first prove (ii)-(iv) under the assumption that

$$
0 \leq e \leq 1 \text { for all } e \in \mathcal{E}_{1} .
$$

(ii) Assume that w.l.o.g.

$$
\alpha:=\sup _{e \in \mathcal{E}_{1}} \bar{i}\left({ }^{\circ} e\right)<\infty .
$$

Let $\varepsilon \in \mathbb{R}_{+}$be given. Define for $e \in \mathcal{E}_{1}, n \in \mathbb{N}$

$$
\mathcal{H}_{e}^{n}:=\left\{g \in \mathcal{E}: e 1_{\{e \geq 1 / n\}} \leq g \leq 2, i(g) \leq \alpha+\varepsilon\right\} .
$$

Then $\mathcal{H}_{e}^{n}$ are internal sets and we show that

$$
\bigcap_{i=1}^{k} \mathcal{H}_{e_{i}}^{n_{i}} \neq \emptyset \text { for all } e_{i} \in \mathcal{E}_{1}, n_{i} \in \mathbb{N} \text { and } k \in \mathbb{N} \text {. }
$$


To this aim let $n:=n_{1} \vee \ldots \vee n_{k}$. As $\mathcal{E}_{1}$ is upwards directed, there exists $\widehat{e} \in \mathcal{E}_{1}$ with $e_{1} \vee \ldots \vee e_{k} \leq \widehat{e}$. Hence

$$
e_{1} 1_{\left\{e_{1} \geq 1 / n_{1}\right\}} \vee \ldots \vee e_{k} 1_{\left\{e_{k} \geq 1 / n_{k}\right\}} \leq \widehat{e} 1_{\{\widehat{e} \geq 1 / n\}} .
$$

As $\widehat{e} \in \mathcal{E}_{1}$, we have $\bar{i}\left({ }^{\circ} \widehat{e}\right) \leq \alpha$ by $(2)$. Hence there exist $1 \geq g_{1} \in \mathcal{E}^{\text {fin }}$ and $1 \geq \delta \in \mathbb{R}_{+}$such that

$$
{ }^{\circ} \widehat{e} \leq g_{1} \text { with }(1+\delta) i\left(g_{1}\right) \leq \alpha+\varepsilon .
$$

Then

$$
\widehat{e} 1_{\{\widehat{e} \geq 1 / n\}} \leq(1+\delta) g_{1}=: g \leq 2 .
$$

Then $2 \geq g \in \mathcal{E}$ and $i(g) \leq \alpha+\varepsilon$ according to (5) and hence $g \in \bigcap_{i=1}^{k} \mathcal{H}_{e_{i}}^{n_{i}}$ by (4) and (6). This proves (3). By compactness there exists $\tilde{e} \in \bigcap_{e \in \mathcal{E}_{1}, n \in \mathbb{N}} \mathcal{H}_{e}^{n}$. Then by Lemma 2

$$
\begin{gathered}
i^{L}\left({ }^{\circ} \tilde{e}\right) \leq{ }^{\circ} i(\tilde{e}) \leq \alpha+\varepsilon, \\
e 1_{\{e \geq 1 / n\}} \leq \tilde{e} \text { for all } e \in \mathcal{E}_{1}, n \in \mathbb{N} .
\end{gathered}
$$

This implies ${ }^{\circ} e \leq{ }^{\circ} \tilde{e}$ for all $e \in \mathcal{E}_{1}$. Hence $\sup _{e \in \mathcal{E}_{1}}{ }^{\circ} e \leq{ }^{\circ} \tilde{e}$ and (7) implies

$$
\bar{i}\left(\sup _{e \in \mathcal{E}_{1}}{ }^{\circ} e\right) \leq \bar{i}\left({ }^{\circ} \tilde{e}\right)=i^{L}\left({ }^{\circ} \tilde{e}\right) \leq \alpha+\varepsilon .
$$

As $\varepsilon \in \mathbb{R}_{+}$was arbitrary, we obtain (ii) under assumption (1).

(iii) follows using Lemma 16 (ii) from

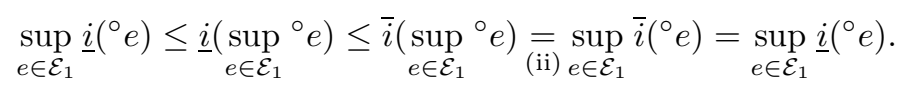

(iv) follows from (ii) and (iii) using Lemma 16 (ii).

Now we prove (i). Here we may assume w.l.o.g. that $\mathcal{E}_{1}$ is upwards directed and (1) holds. Put $f:=\sup _{e \in \mathcal{E}_{1}}{ }^{\circ} e$. We first show that $f$ is $\mathcal{M}(\bar{i})$-measurable. To this aim choose $M_{0} \in \mathcal{M}_{0}$. We show that

$$
1_{M_{0}} \wedge f \in \mathcal{L}(i) .
$$

Then for $0<r$ we have according to Theorem 3 (vi)

$$
\{f>r\} \cap M_{0}=\left\{1_{M_{0}} \wedge f>r\right\} \in \mathcal{M}_{0} .
$$

Hence $f$ is $\mathcal{M}(\bar{i})$-measurable according to Theorem 3 (ii).

To prove (9) let $\varepsilon \in \mathbb{R}_{+}$be given. Choose $h$ with

$$
h \in \mathcal{E}^{f i n}, 1_{M_{0}} \leq h \leq 1, \quad i^{L}\left({ }^{\circ} h\right) \leq{ }^{\circ} i(h) \leq i^{L}\left(M_{0}\right)+\varepsilon .
$$

Then for $e \in \mathcal{E}_{1}$

$$
{ }^{\circ}(e \wedge h) \leq 1_{M_{0}} \wedge{ }^{\circ} e+{ }^{\circ} h-1_{M_{0}} .
$$

Hence according to (iv) and Lemma 1 (v) we have

$$
\begin{aligned}
\bar{i}\left(1_{M_{0}} \wedge f\right) & \leq \bar{i}\left(\sup _{e \in \mathcal{E}_{1}}{ }^{\circ}(e \wedge h)\right) \underset{(\mathrm{iv})}{=} \underline{i}\left(\sup _{e \in \mathcal{E}_{1}}{ }^{\circ}(e \wedge h)\right) \leq \underset{(11)}{\leq} \underline{i}\left(1_{M_{0}} \wedge f\right)+\bar{i}\left({ }^{\circ} h-1_{M_{0}}\right) \\
& \leq \underline{i}\left(1_{M_{0}} \wedge f\right)+\varepsilon .
\end{aligned}
$$

This proves (9). Now we prove that

$$
f \text { is } \mathcal{M}(\underline{i}) \text {-measurable. }
$$


Let $r>0$. Then $\{f>r\} \in \mathcal{M}(\bar{i})$ as just proven. To prove $\{f>r\} \in \mathcal{M}(\underline{i})$ it remains according to Lemma 12 to show:

$$
A \text { internal } \wedge \underline{i}(A)=\infty \Rightarrow \underline{i}(A \cap\{f>r\})=\infty \text { or } \underline{i}(A \cap\{f \leq r\})=\infty .
$$

Assume $\underline{i}(A \cap\{f>r\})<\infty$. Then $\underline{i}\left(A \cap\left\{{ }^{\circ} e>r\right\}\right)<\infty$ for all $e \in \mathcal{E}_{1}$. As ${ }^{\circ} e$ is $\mathcal{M}(\underline{i})$-measurable according to Lemma 15 (ii), we have

$$
\underline{i}\left(A \cap\left\{{ }^{\circ} e \leq r\right\}\right)=\infty .
$$

As $A \cap\left\{{ }^{\circ} e \leq r\right\} \subset A \cap\{e \leq r+1 / n\}$ for each $n \in \mathbb{N}$, we obtain from (14)

$$
\underline{i}(A \cap\{e \leq r+1 / n\})=\infty .
$$

As $\mathcal{C}:=\left\{A \cap\{e \leq r+1 / n\}: e \in \mathcal{E}_{1}, n \in \mathbb{N}\right\}$ is a system of internal sets with the finite intersection property and $|\mathcal{C}| \leq|\widehat{S}|$, we have

$$
\underline{i}\left(A \cap \bigcap_{e \in \mathcal{E}_{1}, n \in \mathbb{N}}\{e \leq r+1 / n\}\right)=\infty
$$

by Lemma 9. Now $\underline{i}(A \cap\{f \leq r\})=\infty$ follows from $A \cap \bigcap_{e \in \mathcal{E}_{1}, n \in \mathbb{N}}\{e \leq r+1 / n\}=$ $A \cap\{f \leq r\}$ and (16), i.e. (13) is proven.

It remains to prove (ii)-(iv) without the assumption (1). It is clear that (ii)-(iv) follows under the assumption

$$
0 \leq e \leq n \text { for all } e \in \mathcal{E}_{1} .
$$

Hence (ii) follows using (i) from

$$
\begin{aligned}
\sup _{g \in \mathcal{E}_{1}} \bar{i}\left({ }^{\circ} g\right) & =\sup _{g \in \mathcal{E}_{1}} \int{ }^{\circ} g d \bar{i}=\sup _{n \in \mathbb{N}} \sup _{g \in \mathcal{E}_{1}} \int{ }^{\circ}(g \wedge n) d \bar{i}=\sup _{n \in \mathbb{N}} \sup _{g \in \mathcal{E}_{1}} \bar{i}\left({ }^{\circ} g \wedge n\right) \\
& =\sup _{n \in \mathbb{N}} \int\left(\sup _{g \in \mathcal{E}_{1}}{ }^{\circ} g\right) \wedge n d \bar{i}=\int \sup _{g \in \mathcal{E}_{1}}{ }^{\circ} g d \bar{i}=\bar{i}\left(\sup _{g \in \mathcal{E}_{1}}{ }^{\circ} g\right) .
\end{aligned}
$$

(iii) follows similary. (iv) follows from (ii) and (iii) using Lemma 16 (ii).

17. Lemma. Let $f_{1}, f_{2}: Y \rightarrow\left[0, \infty\left[\right.\right.$ be $\mathcal{M}(\bar{i})$-measurable and $Y_{0} \subset Y$. Then

$$
\bar{i}\left(f_{1} 1_{Y_{0}}+f_{2} 1_{Y_{0}}\right)=\bar{i}\left(f_{1} 1_{Y_{0}}\right)+\bar{i}\left(f_{2} 1_{Y_{0}}\right) .
$$

Proof. According to Lemma 1 (vii) we may assume that $f_{1}, f_{2}$ are bounded. According to Lemma 1 (iv) it suffices to prove

$$
\bar{i}\left(f_{1} 1_{Y_{0}}+f_{2} 1_{Y_{0}}\right) \geq \bar{i}\left(f_{1} 1_{Y_{0}}\right)+\bar{i}\left(f_{2} 1_{Y_{0}}\right) ;
$$

assume w.l.o.g. that $\bar{i}\left(f_{1} 1_{Y_{0}}+f_{2} 1_{Y_{0}}\right)<\infty$. To prove (1) let $e \in \mathcal{E}^{\text {fin }}$ with

$$
e \geq f_{1} 1_{Y_{0}}+f_{2} 1_{Y_{0}}, e \text { bounded, }
$$

be chosen. According to Lemma $2,{ }^{\circ} e \in \mathcal{L}(i)$ and hence $f_{1} \wedge{ }^{\circ} e,{ }^{\circ} e-f_{1} \wedge{ }^{\circ} e$ are $\mathcal{M}(\bar{i})$-measurable. Therefore $f_{1} \wedge^{\circ} e,{ }^{\circ} e-f_{1} \wedge^{\circ} e \in \mathcal{L}(i)$ (use Theorem $3(\mathrm{v})$ ). Hence using (2)

$$
i^{L}\left({ }^{\circ} e\right)=i^{L}\left(f_{1} \wedge{ }^{\circ} e\right)+i^{L}\left({ }^{\circ} e-f_{1} \wedge^{\circ} e\right) \geq \bar{i}\left(f_{1} 1_{Y_{0}}\right)+\bar{i}\left(f_{2} 1_{Y_{0}}\right) .
$$

Since $i^{L}\left({ }^{\circ} e\right) \leq{ }^{\circ} i(e)$ by Lemma 2 , we obtain (1).

18. Lemma. Let $f_{n}: Y \rightarrow\left[0, \infty\left[\right.\right.$ be $\mathcal{M}(\underline{i})$-measurable and $Y_{0} \subset Y$. Then

(i) $\underline{i}\left(f_{1} 1_{Y_{0}}+f_{2} 1_{Y_{0}}\right)<\infty \Rightarrow \underline{i}\left(f_{1} 1_{Y_{0}}+f_{2} 1_{Y_{0}}\right)=\underline{i}\left(f_{1} 1_{Y_{0}}\right)+\underline{i}\left(f_{2} 1_{Y_{0}}\right)$;

(ii) $f_{n} \uparrow f \in \mathbb{R}^{Y} \wedge \underline{i}\left(f 1_{Y_{0}}\right)<\infty \Rightarrow \underline{i}\left(f_{n} 1_{Y_{0}}\right) \uparrow \underline{i}\left(f 1_{Y_{0}}\right)$. 
Proof. (i) According to Lemma 1 (iv) it suffices to prove

$$
\underline{i}\left(f_{1} 1_{Y_{0}}+f_{2} 1_{Y_{0}}\right) \leq \underline{i}\left(f_{1} 1_{Y_{0}}\right)+\underline{i}\left(f_{2} 1_{Y_{0}}\right) .
$$

Let $e \in \mathcal{E}^{\text {fin }}$ with

$$
0 \leq e \leq f_{1} 1_{Y_{0}}+f_{2} 1_{Y_{0}}
$$

be given. Then we have

$$
{ }^{\circ} e \leq f_{1} \wedge{ }^{\circ} e+f_{2} \wedge{ }^{\circ} e, \quad f_{1} \wedge{ }^{\circ} e \leq f_{1} 1_{Y_{0}}, \quad f_{2} \wedge{ }^{\circ} e \leq f_{2} 1_{Y_{0}} .
$$

Hence

$$
i^{L}\left({ }^{\circ} e\right) \leq i^{L}\left(f_{1} \wedge{ }^{\circ} e\right)+i^{L}\left(f_{2} \wedge^{\circ} e\right) \leq \underline{i}\left(f_{1} 1_{Y_{0}}\right)+\underline{i}\left(f_{2} 1_{Y_{0}}\right) .
$$

As $\underline{i}\left(f_{1} 1_{Y_{0}}+f_{2} 1_{Y_{0}}\right)<\infty$, we obtain from (2) and Lemma 6 that $i^{L}\left({ }^{\circ} e\right)={ }^{\circ} i(e)$. This implies (1).

(ii) We have to prove

$$
\lim _{n \rightarrow \infty} \underline{i}\left(f_{n} 1_{Y_{0}}\right) \geq \underline{i}\left(f 1_{Y_{0}}\right)
$$

Choose $e_{n} \in \mathcal{E}^{\text {fin }}$ with

$$
e_{n} \leq e_{n+1}, e_{n} \leq f 1_{Y_{0}},{ }^{\circ} i\left(e_{n}\right) \uparrow \underline{i}\left(f 1_{Y_{0}}\right) .
$$

As $\underline{i}\left(f 1_{Y_{0}}\right)<\infty$, we obtain by Lemma 6 that ${ }^{\circ} i\left(e_{n}\right)=i^{L}\left({ }^{\circ} e_{n}\right)$ and hence

$$
\begin{aligned}
\underline{i}\left(f 1_{Y_{0}}\right) & =\lim _{n \rightarrow \infty}{ }^{\circ} i\left(e_{n}\right)=\lim _{n \rightarrow \infty} i^{L}\left({ }^{\circ} e_{n}\right)=i^{L}\left(\lim _{n \rightarrow \infty}{ }^{\circ} e_{n}\right) \\
& =i^{L}\left(\lim _{n \rightarrow \infty}\left({ }^{\circ} e_{n} \wedge f_{n}\right)\right)=\lim _{n \rightarrow \infty} i^{L}\left({ }^{\circ} e_{n} \wedge f_{n}\right) \leq \lim _{n \rightarrow \infty} \underline{i}\left(f_{n} 1_{Y_{0}}\right) .
\end{aligned}
$$

Proof of Theorem 7. $\bar{i} \mid \mathcal{M}(\bar{i}) \cap Y_{0}$ is a measure according to Lemma 17 and Lemma 1 (vii). $\bar{i}\left(f 1_{Y_{0}}\right)=\int f_{\mid Y_{0}} d \bar{i}_{\mid Y_{0}}$ follows for $\mathcal{M}(\bar{i})$-elementary functions by Lemma 17 and Lemma 1 (ii). The general case follows then from Lemma 1 (vii).

It remains to prove

$$
\begin{gathered}
\underline{i} \mid \mathcal{M}(\underline{i}) \cap Y_{0} \text { is a measure, } \\
\underline{i}\left(f 1_{Y_{0}}\right)=\left.\left.\int f\right|_{Y_{0}} d \underline{i}\right|_{Y_{0}} .
\end{gathered}
$$

$\operatorname{Ad}(1)$ : Let $M_{1} \cap Y_{0}$ and $M_{2} \cap Y_{0}$ be disjoint with $M_{1}, M_{2} \in \mathcal{M}(\underline{i})$. If $\underline{i}\left(\left(M_{1} \cap Y_{0}\right) \cup\left(M_{2} \cap Y_{0}\right)\right)<\infty$, then

$$
\underline{i}\left(\left(M_{1} \cap Y_{0}\right) \cup\left(M_{2} \cap Y_{0}\right)\right)=\underline{i}\left(M_{1} \cap Y_{0}\right)+\underline{i}\left(M_{2} \cap Y_{0}\right)
$$

by Lemma 18 (i). To prove that $\underline{i} \mid \mathcal{M}(\underline{i}) \cap Y_{0}$ is additive it suffices therefore to show

$$
\underline{i}\left(M_{1} \cap Y_{0}\right)<\infty, \underline{i}\left(M_{2} \cap Y_{0}\right)<\infty \Rightarrow \underline{i}\left(\left(M_{1} \cap Y_{0}\right) \cup\left(M_{2} \cap Y_{0}\right)\right)<\infty .
$$

As $M_{1} \in \mathcal{M}(\underline{i})$ we have with $A:=Y_{0} \cap\left(M_{1} \cup M_{2}\right)$ :

$$
\underline{i}(A)=\underline{i}\left(A \cap M_{1}\right)+\underline{i}\left(A \backslash M_{1}\right) \leq \underline{i}\left(Y_{0} \cap M_{1}\right)+\underline{i}\left(Y_{0} \cap M_{2}\right)<\infty,
$$

i.e. (3) holds.

Now let $\mathcal{M}(\underline{i}) \ni M_{n} \uparrow M_{\infty}$ be given. To prove (1), it suffices to show

$$
\underline{i}\left(M_{n} \cap Y_{0}\right) \uparrow \underline{i}\left(M_{\infty} \cap Y_{0}\right) \text {. }
$$

If $\underline{i}\left(M_{\infty} \cap Y_{0}\right)<\infty$, we obtain (4) from Lemma 18 (ii). Therefore, let $\underline{i}\left(M_{\infty} \cap Y_{0}\right)=$ $\infty$ and assume indirectly that

$$
\lim _{n \rightarrow \infty} \underline{i}\left(M_{n} \cap Y_{0}\right) \leq c_{1} \text { for some } c_{1} \in \mathbb{R} .
$$


We consider the following two cases:

$$
\begin{aligned}
& \sup \left\{\underline{i}(T): T \subset M_{\infty} \cap Y_{0}, \quad \underline{i}(T)<\infty\right\}=\infty, \\
& \sup \left\{\underline{i}(T): T \subset M_{\infty} \cap Y_{0}, \quad \underline{i}(T)<\infty\right\}<\infty .
\end{aligned}
$$

Assume that (6) holds. Hence according to Lemma 7 (ii) we obtain

$$
\sup \left\{\underline{i}\left(M_{0}\right): M_{0} \subset M_{\infty} \cap Y_{0}, M_{0} \in \mathcal{M}_{0}\right\}=\infty .
$$

Choose $M_{0} \in \mathcal{M}_{0}$ with $M_{0} \subset M_{\infty} \cap Y_{0}$ and $i^{L}\left(M_{0}\right)>c_{1}$. As $M_{0} \cap M_{n} \in \mathcal{M}_{0}$ and $M_{0} \cap M_{n} \uparrow M_{0} \cap M_{\infty}=M_{0}$, we obtain the following contradiction:

$$
\begin{aligned}
c_{1}<i^{L}\left(M_{0}\right) & =\lim _{n \rightarrow \infty} i^{L}\left(M_{0} \cap M_{n}\right)=\lim _{n \rightarrow \infty} \underline{i}\left(M_{0} \cap M_{n}\right) \\
& \leq \lim _{n \rightarrow \infty} \underline{i}\left(Y_{0} \cap M_{n}\right) \leq c_{1} .
\end{aligned}
$$

Assume that (7) holds. We construct sets $C_{n}$ with

$$
\left\{\begin{array}{l}
C_{n} \text { internal, } \quad C_{n} \subset M_{\infty} \cap Y_{0} \backslash M_{n} \cap Y_{0}, \\
C_{n} \downarrow, \quad \underline{i}\left(C_{n}\right)=\infty
\end{array}\right.
$$

Since $C_{n}$ by (8) are internal with $\bigcap_{n=1}^{\infty} C_{n}=\emptyset$, we obtain $C_{n}=\emptyset$ for some $n \in$ $\mathbb{N}$, contradicting $\underline{i}\left(C_{n}\right)=\infty$. Thus it remains to construct $C_{n}$ fulfilling (8). As $\underline{i}\left(M_{\infty} \cap Y_{0} \backslash M_{1} \cap Y_{0}\right)=\infty$ we obtain an internal set $C_{1} \subset M_{\infty} \cap Y_{0} \backslash M_{1} \cap Y_{0}$ with $\underline{i}\left(C_{1}\right)=\infty$ by Lemma 10 . Now assume inductively that internal sets $C_{1} \supset \ldots \supset C_{n}$ with $C_{j} \subset M_{\infty} \cap Y_{0} \backslash M_{j} \cap Y_{0}$ and $\underline{i}\left(C_{j}\right)=\infty$ are constructed. As $M_{\infty} \backslash M_{n+1} \in \mathcal{M}(\underline{i})$ we have

$\infty=\underline{i}\left(C_{n}\right)=\underline{i}\left(C_{n} \cap Y_{0}\right)=\underline{i}\left(\left(C_{n} \cap Y_{0}\right) \cap\left(M_{\infty} \backslash M_{n+1}\right)\right)+\underline{i}\left(\left(C_{n} \cap Y_{0}\right) \backslash\left(M_{\infty} \backslash M_{n+1}\right)\right)$.

Since

$$
\underline{i}\left(\left(C_{n} \cap Y_{0}\right) \backslash\left(M_{\infty} \backslash M_{n+1}\right)\right) \leq \underline{i}\left(M_{n+1} \cap Y_{0}\right)<\infty,
$$

we obtain $\underline{i}\left(\left(C_{n} \cap Y_{0}\right) \cap\left(M_{\infty} \backslash M_{n+1}\right)\right)=\infty$. Hence by Lemma 10 there exists an internal set $C_{n+1} \subset\left(C_{n} \cap Y_{0}\right) \cap\left(M_{\infty} \backslash M_{n+1}\right)$ with $\underline{i}\left(C_{n+1}\right)=\infty$. This proves (8) for $n+1$. Hence (1) is proven.

$\operatorname{Ad}(2)$ : If $f=1_{M}$ with $M \in \mathcal{M}(\underline{i})$, then (2) follows by definition of $\left.\underline{i}\right|_{Y_{0}}$. Now let $0 \leq f$ be elementary. Then $f=\sum_{j=1}^{n} \alpha_{j} 1_{M_{j}}$ with disjoint $M_{j} \in \mathcal{M}(\underline{i})$ and $\alpha_{j}>0$ given. If $\underline{i}\left(f 1_{Y_{0}}\right)<\infty$, then (2) follows from Lemma 18 (i) and Lemma 1 (ii). Now let $\underline{i}\left(f 1_{Y_{0}}\right)=\infty$ and $\alpha:=\max _{j=1}^{n} \alpha_{j}$. Then $\underline{i}\left(\alpha 1_{\sum_{j=1}^{n} M_{j} \cap Y_{0}}\right)=\infty$ and hence $\underline{i}\left(1_{\sum_{j=1}^{n} M_{j} \cap Y_{0}}\right)=\infty$. Hence by (1) there exists $j_{0}$ with $\underline{i}\left(M_{j_{0}} \cap Y_{0}\right)=$ $\infty$. Therefore $\infty=\sum_{j=1}^{n} \alpha_{j} \underline{i}\left(M_{j} \cap Y_{0}\right)=\left.\left.\int f\right|_{Y_{0}} d \underline{i}\right|_{Y_{0}}$. This proves (2) for $\mathcal{M}(\underline{i})$ elementary functions. Using Lemma 18 (ii) we obtain (2) for $\mathcal{M}(\underline{i})$-measurable functions $f \geq 0$ if $\underline{i}\left(f 1_{Y_{0}}\right)<\infty$.

Now consider the case $\underline{i}\left(f 1_{Y_{0}}\right)=\infty$. We first show

$$
\underline{i}\left([f \wedge n] 1_{Y_{0}}\right) \uparrow \infty .
$$

Assume indirectly that

$$
c:=\sup \underline{i}\left([f \wedge n] 1_{Y_{0}}\right)<\infty .
$$

Let $e \leq f 1_{Y_{0}}$ with $e \in \mathcal{E}^{f i n}$ given. Then there exists $n \in \mathbb{N}$ with $e \leq n$ as $e$ is internal. Hence

$$
e \leq[f \wedge n] 1_{Y_{0}} .
$$


From (11) we obtain that

$$
{ }^{\circ} i(e) \leq \underline{i}\left([f \wedge n] 1_{Y_{0}}\right) \leq c .
$$

Hence $\underline{i}\left(f 1_{Y_{0}}\right) \leq c$, contradicting our assumption $\underline{i}\left(f 1_{Y_{0}}\right)=\infty$. Therefore (9) holds. Consequently it suffices to show (2) for $\mathcal{M}(\underline{i})$-measurable function $f$ with $0 \leq f \leq 1$ and $\underline{i}\left(f 1_{Y_{0}}\right)=\infty$. Hence we have to show that $\left.\int f_{\mid Y_{0}} d \underline{i}\right|_{Y_{0}}=\infty$. Let indirectly

$$
c:=\int f_{\mid Y_{0}} d \underline{i}_{\mid Y_{0}}<\infty
$$

Then

$$
c=\sum_{n=1}^{\infty} \int f 1_{\left\{\frac{1}{n+1}<f \leq \frac{1}{n}\right\} \cap Y_{0}} d \underline{i}_{\mid Y_{0}} .
$$

Now we have by (12)

$$
\underline{i}\left(\left\{\frac{1}{n+1}<f \leq \frac{1}{n}\right\} \cap Y_{0}\right) \leq(n+1) \int f_{\mid Y_{0}} d \underline{i}_{\mid Y_{0}}<\infty .
$$

Hence according to Lemma 7 (ii) there exists $M_{n} \in \mathcal{M}_{0}$ with $M_{n} \subset\left\{\frac{1}{n+1}<f \leq\right.$ $\left.\frac{1}{n}\right\} \cap Y_{0}$ and $\underline{i}\left(M_{n}\right)=\underline{i}\left(\left\{\frac{1}{n+1}<f \leq \frac{1}{n}\right\} \cap Y_{0}\right)<\infty$. Therefore according to (1)

$$
\left.\underline{i}\right|_{Y_{0}}\left(\left\{\frac{1}{n+1}<f \leq \frac{1}{n}\right\} \cap Y_{0}-M_{n}\right)=0 .
$$

Put $M_{\infty}:=\biguplus_{n=1}^{\infty} M_{n}$; then from (1) and (13) we obtain

$$
\underline{i}_{\mid Y_{0}}\left(\{f>0\} \cap Y_{0} \backslash M_{\infty}\right)=0 .
$$

Hence

$$
\underline{i}\left(f 1_{Y_{0}} 1_{\{f>0\}}-f 1_{M_{\infty}}\right) \leq 1 \cdot \underline{i}\left(Y_{0} \cap\{f>0\}-M_{\infty}\right)=0 .
$$

From (15), Lemma 1 (v), Lemma 1 (vii) and (12) we obtain

$$
\begin{aligned}
\underline{i}\left(f 1_{Y_{0}}\right) & =\underline{i}\left(f 1_{Y_{0}}-f 1_{M_{\infty}}+f 1_{M_{\infty}}\right) \leq \underline{i}\left(f 1_{Y_{0}}-f 1_{M_{\infty}}\right)+\bar{i}\left(f 1_{M_{\infty}}\right) \\
& =\lim _{n \rightarrow \infty} \bar{i}\left(f 1_{\cup_{i=1}^{n} M_{i}}\right)=\lim _{n \rightarrow \infty} \underline{i}\left(f 1_{\bigcup_{i=1}^{n} M_{i}}\right) \\
& =\lim _{n \rightarrow \infty} \int f 1_{\cup_{i=1}^{n} M_{i}} d \underline{i}_{\mid Y_{0}} \leq \int f 1_{Y_{0}} d \underline{i}_{Y_{0}}<\infty .
\end{aligned}
$$

This contradicts our assumption $\underline{i}\left(f 1_{Y_{0}}\right)=\infty$.

Proof of Theorem 8. (i) It suffices to prove

$$
\alpha:=\underline{i}\left(\sup _{e \in \mathcal{E}_{1}}{ }^{\circ} e 1_{Y_{0}}\right) \leq \sup _{e \in \mathcal{E}_{1}} \underline{i}\left({ }^{\circ} e 1_{Y_{0}}\right) .
$$

By Theorem 7 (ii) we may assume that $0 \leq e \leq 1$ for $e \in \mathcal{E}_{1}$. Now let $\varepsilon \in \mathbb{R}_{+}$be given. As $\alpha$ is finite, there exists $g \in \mathcal{E}^{\text {fin }}$ with

$$
\begin{gathered}
g \leq\left(\sup _{e \in \mathcal{E}_{1}}{ }^{\circ} e\right) 1_{Y_{0}}, \\
i^{L}\left({ }^{\circ} g\right)={ }^{\circ} i(g) \geq \alpha-\varepsilon .
\end{gathered}
$$

Hence by (2), (1) and Theorem 6 (iii) we have

$$
\begin{aligned}
\alpha-\varepsilon & \left.\leq \underline{i}\left({ }^{\circ} g\right) \quad=\underline{i}^{\circ} g \wedge \sup _{e \in \mathcal{E}_{1}} e\right)=\underline{i}\left(\sup _{e \in \mathcal{E}_{1}}{ }^{\circ}(g \wedge e)\right) \\
& =\sup _{e \in \mathcal{E}_{1}} \underline{i}\left({ }^{\circ}(g \wedge e)\right) \leq \sup _{e \in \mathcal{E}_{1}} \underline{i}\left({ }^{\circ} e 1_{Y_{0}}\right) .
\end{aligned}
$$

As $\varepsilon \in \mathbb{R}_{+}$was arbitrary, we obtain the assertion. 
(ii) It suffices to show

$$
\beta:=\bar{i}\left(\left(\sup _{e \in \mathcal{E}_{1}}{ }^{\circ} e\right) 1_{Y_{0}}\right) \leq \sup _{e \in \mathcal{E}_{1}} \bar{i}\left({ }^{\circ} e 1_{Y_{0}}\right) .
$$

According to Theorem 7 (iii) we may assume that $0 \leq e \leq 1$ for $e \in \mathcal{E}_{1}$.

As $\beta$ is finite, there exists $g$ with

$$
g \in \mathcal{E}^{f i n}, \sup _{e \in \mathcal{E}_{1}}{ }^{\circ} e \cdot 1_{Y_{0}} \leq g \leq 1
$$

Then

$$
\sup _{e \in \mathcal{E}_{1}}{ }^{\circ} e \wedge^{\circ} g=\sup _{e \in \mathcal{E}_{1}}{ }^{\circ}(e \wedge g) \in \mathcal{L}(i)
$$

according to Theorem 6 (iv). Then according to Theorem 6 there exists a sequence $e_{n} \in \mathcal{E}_{1}$ with $e_{n} \wedge g \leq e_{n+1} \wedge g$ and

$$
\infty>\bar{i}\left(\sup _{e \in \mathcal{E}_{1}}{ }^{\circ}(e \wedge g)\right)=\lim _{n \rightarrow \infty} \bar{i}\left({ }^{\circ}\left(e_{n} \wedge g\right)\right)=\bar{i}\left(\sup _{n \in \mathbb{N}}{ }^{\circ}\left(e_{n} \wedge g\right)\right)
$$

Hence $\sup _{e \in \mathcal{E}_{1}}{ }^{\circ}(e \wedge g)=\sup _{n \in \mathbb{N}}{ }^{\circ}\left(e_{n} \wedge g\right) \bar{i}$-a.e., and this implies according to Theorem 7

$$
\begin{aligned}
\bar{i}\left(\sup _{e \in \mathcal{E}_{1}} e e 1_{Y_{0}}\right) & =\bar{i}\left(\sup _{e \in \mathcal{E}_{1}}{ }^{\circ}(e \wedge g) 1_{Y_{0}}\right)=\bar{i}\left(\sup _{n \in \mathbb{N}}{ }^{\circ}\left(e_{n} \wedge g\right) 1_{Y_{0}}\right) \\
& =\lim _{n \rightarrow \infty} \bar{i}\left({ }^{\circ}\left(e_{n} \wedge g\right) 1_{Y_{0}}\right) \leq \sup _{e \in \mathcal{E}_{1}} \bar{i}\left({ }^{\circ} e 1_{Y_{0}}\right) .
\end{aligned}
$$
19. Example. We show that in Theorem 8 (ii) the assumption $\bar{i}\left(\sup _{e \in \mathcal{E}_{1}}{ }^{\circ} e 1_{Y_{0}}\right)<\infty$
cannot be dispensed with:

To this aim we construct an internal content $\nu: \mathcal{R} \rightarrow{ }^{*}[0, \infty[$ on a ring $\mathcal{R} \subset P(Y)$ and choose $\mathcal{E}$ and $i=i_{\nu}$ according to Example 5. We furthermore construct $\mathcal{D} \subset \mathcal{R}$ with cardinality of $\mathcal{D}$ smaller than or equal to $\widehat{S}, \mathcal{D} \uparrow$ and $Y_{0} \subset Y$ such that

$$
\bar{\nu}\left(D \cap Y_{0}\right)=0 \text { for } D \in \mathcal{D}, \quad \bar{\nu}\left(\bigcup_{D \in \mathcal{D}} D \cap Y_{0}\right)=\infty .
$$

Choosing $\mathcal{E}_{1}:=\left\{1_{D}: D \in \mathcal{D}\right\}$ we obtain

$$
\infty=\bar{i}\left(\sup _{e \in \mathcal{E}_{1}}{ }^{\circ} e 1_{Y_{0}}\right) \neq \sup _{e \in \mathcal{E}_{1}} \bar{i}\left({ }^{\circ} e 1_{Y_{0}}\right)=0 .
$$

Let $Y:={ }^{*} \mathbb{R} \times{ }^{*} \mathbb{R}$ and denote by $\lambda$ the Lebesgue-measure on the Borel- $\sigma$-algebra $\mathbb{B}$ and by $Z$ the counting measure on $\mathbb{B}$. Then

$$
\mu(B):=\int \lambda\left(B_{x}\right) Z(d x) \text { for } B \in \mathbb{B} \times \mathbb{B}
$$

defines a measure on $\mathbb{B} \times \mathbb{B}$. Consider

$$
\mathcal{R}={ }^{*}\left\{\bigcup_{x \in E}\{x\} \times B: E \subset \mathbb{R} \text { finite and } B \in \mathbb{B} \text { bounded }\right\}
$$

Then $\nu={ }^{*} \mu \mid \mathcal{R}$ is an internal content on a ring with values in $*[0, \infty[$. Put $\mathcal{D}=$ $\left\{\bigcup_{x \in E}\{x\} \times{ }^{*}[-n, n]: E \subset \mathbb{R}\right.$ finite, $\left.n \in \mathbb{N}\right\}$. Then $\mathcal{D} \uparrow$ and the cardinality of $\mathcal{D} \subset \mathcal{R}$ is smaller than or equal to $\widehat{S}$. Put

$$
Y_{0}:=\left\{(x, y) \in \mathbb{R} \times{ }^{*} \mathbb{R}: y \approx x\right\} .
$$


Then $D \cap Y_{0} \subset\left\{(x, y) \in E \times{ }^{*} \mathbb{R}: y \approx x\right\}$ for some finite $E \subset \mathbb{R}$ and $\bigcup_{D \in \mathcal{D}} D \cap Y_{0}=$ $\{(x, y) \in \mathbb{R} \times * \mathbb{R}: y \approx x\}=Y_{0}$.

Now it suffices to show:

$$
\begin{gathered}
\bar{\nu}\left(\left\{(x, y): x \approx y \in{ }^{*} \mathbb{R}\right\}\right)=0 \text { for all } x \in \mathbb{R} \\
\bar{\nu}\left(Y_{0}\right)=\infty
\end{gathered}
$$

Ad (1): We have $\left\{(x, y): x \approx y \in{ }^{*} \mathbb{R}\right\} \subset\{x\} \times{ }^{*}\left[x-\frac{1}{n}, x+\frac{1}{n}\right]$ for all $n \in \mathbb{N}$. Then $\bar{\nu}\left(\{x\} \times^{*}[x-1 / n, x+1 / n]\right)=s t^{*} \mu\left(\{x\} \times *\left[x-\frac{1}{n}, x+\frac{1}{n}\right]\right)=\mu\left(\{x\} \times\left[x-\frac{1}{n}, x+\frac{1}{n}\right]\right)=$ $\frac{2}{n} \rightarrow_{n \rightarrow \infty} 0$. This proves (1).

$\operatorname{Ad}(2)$ : Let $B \in \mathcal{R}$ with $Y_{0} \subset B$ be given. We have to show

$$
{ }^{\circ} \nu(B)=\infty \text {. }
$$

For $x \in \mathbb{R}$ we have $m(x):=\left\{y \in{ }^{*} \mathbb{R}: y \approx x\right\} \subset B_{x}$. As $B_{x}$ is internal, there exists for each $x \in \mathbb{R}$ - according to the permanence principle $-n_{x} \in \mathbb{N}$ with

$$
{ }^{*}\left[x-\frac{1}{n_{x}}, x+\frac{1}{n_{x}}\right] \subset B_{x} .
$$

Hence there exists $n_{0} \in \mathbb{N}$ such that

$$
I_{0}=\left\{x \in \mathbb{R}: n_{x}=n_{0}\right\} \text { is uncountable. }
$$

Therefore according to (4)

$$
\biguplus_{x \in I_{0}}\{x\} \times^{*}\left[x-\frac{1}{n_{0}}, x+\frac{1}{n_{0}}\right] \subset B .
$$

Thus for each finite $E \subset I_{0}$ we have

$$
{ }^{\circ} \nu(B) \geq \sum_{x \in E}{ }^{\circ} \nu\left(\{x\} \times{ }^{*}\left[x-1 / n_{0}, x+1 / n_{0}\right]\right)=|E| \frac{2}{n_{0}} .
$$

As this holds for each finite $E \subset I_{0}$, we obtain (3).

The following example shows that the results of Theorem 4 cannot be improved. We consider a set function $\nu$ as in Example 5.

20. Example. (i) Let $Y:=\{1,2,3\}$. Put $\mathcal{R}:=\{\emptyset,\{1,2\},\{3\}, Y\}$. Let $h \in{ }^{*} \mathbb{N} \backslash \mathbb{N}$ and put

$$
\nu(\emptyset)=0, \nu(\{1,2\})=\nu(\{3\})=h, \nu(Y)=2 h .
$$

Then $\nu: \mathcal{R} \rightarrow{ }^{*}[0, \infty[$ is an internal content with

$$
\mathcal{M}(\underline{\nu})=\{\emptyset,\{3\},\{1,2\}, Y\} \text { and } \mathcal{M}(\bar{\nu})=\mathcal{P}(Y) .
$$

As $\mathcal{M}_{0}=\{\emptyset\}$ we have

$\underline{\nu} \mid \mathcal{M}(\underline{\nu})$ is not a saturated measure;

$$
\mathcal{M}(\underline{\nu}) \underset{\neq}{\subset} \mathcal{M}(\bar{\nu}) .
$$


(ii) Let $Y \neq \emptyset, \mathcal{R}:=\{\emptyset\}$ and $\nu(\emptyset)=0$. Then $\nu$ is an internal content where $\underline{\nu}(A)=0$ for each $A \subset Y$ and $\bar{\nu}(A)=\infty$ for each $\emptyset \neq A \subset Y$. Therefore $\mathcal{M}(\underline{\nu})=$ $\mathcal{M}(\bar{\nu})=\mathcal{P}(Y)$ and $\mathcal{M}_{0}=\{\emptyset\}$. Hence

$$
\mathcal{M}_{0} \underset{\neq}{\subset}\{A \in \mathcal{M}(\underline{\nu}): \underline{\nu}(A) \in \mathbb{R}\}(=\mathcal{P}(Y))
$$

and therefore

$$
\mathcal{L}\left(i_{\nu}\right) \underset{\neq}{\subset}\left\{f \in \mathbb{R}^{Y}: f \text { is } \underline{i_{\nu}} \mid \mathcal{M}\left(\underline{i_{\nu}}\right) \text {-Lebesgue-integrable }\right\} .
$$

\section{REFERENCES}

[1] Aldaz, J. M., A modified functional approach to nonstandard measure theory, Caribb. J. Math. Comput. Sci. 3 (1993), 17-20. MR96g:28026

[2] Cutland, N., Nonstandard Analysis and its Applications, London Mathematical Society Student Texts, 10, Cambridge University Press, Cambridge (1988). MR89m:03060

[3] Floret, K., Maß- und Integrationstheorie, B. G. Teubner, Stuttgart, 1981. MR:82m:28001

[4] Hurd, A. and Loeb, P., An introduction to nonstandard real analysis, Academic Press, Orlando, Tokyo (1985). MR87d:03184

[5] Landers, D. and Rogge, L., Universal Loeb-measurability of sets and of the standard part map with applications, Trans. Amer. Math. Soc. 304 (1987), 229-243. MR89d:28015

[6] Landers, D. and Rogge, L., Nonstandard methods for families of $\tau$-smooth probability measures, Proc. Amer. Math. Soc. 103 (1988), 1152-1156. MR89j:28007

[7] Landers, D. and Rogge, L., Nichtstandard Analysis, Springer-Verlag, Berlin, New York, Tokyo, 1994. MR95i:03140

[8] Loeb, P., Conversion from nonstandard to standard measure spaces and applications in probability theory, Trans. Amer. Math. Soc. 211 (1975), 113-122. MR52:10980

[9] Loeb, P., Weak limits of measures and the standard part map, Proc. Amer. Math. Soc. 77 (1979), 128-135. MR80i:28020

[10] Loeb, P., A functional approach to nonstandard measure theory, Amer. Math. Soc. Contemporary Math. 26 (1984), 251-261. MR86b:28026

[11] Loeb, P., A nonstandard functional approach to Fubini's theorem, Proc. Amer. Math. Soc. 93 (1985), 343-346. MR86f:28026

[12] Pfeffer, W., Integrals and measures, Marcel Dekker, Inc., New York, Basel, 1977. MR57:573

[13] Sommers, U., Theorie unendlicher Loeb-Maße, Diplomarbeit, Duisburg, 1998.

Mathematisches Institut der Universität zu Köln, Weyertal 86, D-50931 Köln, GerMANY

E-mail address: landers@mi.uni-koeln.de

Fachbereich Mathematik der Gerhard-Mercator-Universität GHS Duisburg, LotharStrasse 65, D-47048 Duisburg, Germany

E-mail address: rogge@math.uni-duisburg.de 